\title{
Tyrosine 136 phosphorylation of a-synuclein aggregates in the Lewy body dementia brain: involvement of serine 129 phosphorylation by casein kinase 2
}

Kazunori Sano ${ }^{1^{*} \mathbb{D}}$, Yasushi Iwasaki ${ }^{2}$, Yuta Yamashita ${ }^{1}$, Keiichi Irie ${ }^{1}$, Masato Hosokawa ${ }^{3}$, Katsuya Satoh ${ }^{4}$ and Kenichi Mishima'

\begin{abstract}
Serine 129 (S129) phosphorylation of a-synuclein (aSyn) is a central feature of Lewy body (LB) disease pathology. Although the neighboring tyrosine residues $\mathrm{Y} 125, \mathrm{Y} 133$, and $\mathrm{Y} 136$ are also phosphorylation sites, little is known regarding potential roles of phosphorylation cross-talk between these sites and its involvement in the pathogenesis of LB disease. Here, we found that aSyn aggregates are predominantly phosphorylated at Y136 in the Lewy body dementia brain, which is mediated by unexpected kinase activity of Casein kinase 2 (CK2). Aggregate formation with $\mathrm{S} 129$ and $\mathrm{Y} 136$ phosphorylation of recombinant aSyn (r-aSyn) were induced by CK2 but abolished by replacement of S129 with alanine (S129A) in vitro. Mutation of Y136 to alanine (Y136A) promoted aggregate formation and S129 phosphorylation of r-aSyn by CK2 in vitro. Introduction of Y136A r-aSyn oligomers into cultured cells exhibited increased levels of aggregates with S129 phosphorylation compared to wild-type r-aSyn oligomers. In addition, aggregate formation with S129 phosphorylation induced by introduction of wild-type r-aSyn oligomers was significantly attenuated by CK2 inhibition, which resulted in an unexpected increase in Y136 phosphorylation in cultured cells. Our findings suggest the involvement of CK2-related aSyn Y136 phosphorylation in the pathogenesis of LB disease and its potential as a therapeutic target.
\end{abstract}

Keywords: a-Synuclein, Lewy body dementia, Y136 phosphorylation, S129 phosphorylation, Casein kinase 2

\section{Introduction}

Lewy body (LB) diseases, including Lewy body dementia (LBD) and Parkinson's disease (PD), are progressive diseases characterized by extensive accumulation of intracellular proteinaceous inclusions composed mainly of aggregated $\alpha$-synuclein ( $\alpha$ Syn) in the brain called LB. Although the pathological mechanisms of LB disease

\footnotetext{
*Correspondence: ksano@fukuoka-u.ac.jp

${ }^{1}$ Department of Physiology and Pharmacology, Faculty of Pharmaceutical

Sciences, Fukuoka University, 8-19-1 Nanakuma, Jonan-ku,

Fukuoka 814-0180, Japan

Full list of author information is available at the end of the article
}

have not been fully elucidated, accumulation of LB, i.e., $\alpha$ Syn aggregates, is thought to play a significant role.

A significant proportion of $\alpha$ Syn accumulated within LB is phosphorylated on the C-terminal serine 129 (S129), while only a small fraction of $\alpha$ Syn is constitutively phosphorylated at this residue in the brain without LB pathology. Earlier in vitro and vivo studies yielded contrasting results regarding the significance of S129 phosphorylation (pS129) for LB formation, showing facilitatory [10,32], inhibitory [6, 24], or no effect [17, 30] of phosphorylation on $\alpha$ Syn aggregation. In mice inoculated with recombinant $\alpha$ Syn ( $r-\alpha$ Syn) fibrils, the S129-phosphorylated and nonphosphorylated forms were shown 
to cause prion-like seeding and accumulation of endogenous $\alpha$ Syn in the brain, and the phosphorylated form showed greater potency to induce the pathology than the nonphosphorylated form [14]. Therefore, although pS129 is not necessarily required for LB formation, it appears to induce more severe pathology.

aSyn tyrosines Y125, Y133, and Y136 are located in close proximity to S129 at the C-terminus (Fig. 1a), which raises questions regarding whether these tyrosine residues are phosphorylated and whether there are interactions among the phosphorylated residues, including S129 and its association with $\alpha$ Syn aggregate formation. Human $r-\alpha$ Syn incubated with spleen tyrosine kinase (Syk) [22] and $\alpha$ Syn in pervanadate-treated cultured human cells [8] have been reported to be phosphorylated at Y125, Y133, and Y136. Several studies have demonstrated the presence of $\alpha$ Syn phosphorylated at Y125 (pY125) in the human brain. An immunohistochemical study identified pY125 within LB in a case of familial PD with G51D mutation [15]. Immunoblotting analysis detected pY125 at similar levels in PD and control brains [19] but at higher levels in control than LBD brains [7]. Other studies showed that pY125 is not a component of LB in LBD and PD by immunohistochemical and immunoblotting analyses [9] and mass spectrometry [1]. pY125 has been reported to inhibit toxic oligomer formation of $\alpha$ Syn in Drosophila [7], whereas an in vitro study showed that pY125 had no influence on aggregation of synthetic $\alpha$ Syn $[11,30]$. Therefore, $\alpha$ Syn appears to be phosphorylated at Y125 in the human brain, but the role of pY125 in $\alpha$ Syn aggregate formation has not been fully elucidated. Immunoblotting analysis indicated the presence of phosphorylated Y133 (pY133) at similar levels in LBD, PD, and control brains [9], suggesting that pY133 may not be crucial for LB pathology. In contrast, there have been few studies regarding the presence of phosphorylated Y136 (pY136) in the human brain and its physiological roles and implications in the pathogenesis of LB disease.

Casein kinase (CK) 1 and 2 are ubiquitous serine/ threonine protein kinases expressed in all eukaryotes. Although both CK1 and CK2 have been shown to constitutively phosphorylate S129 of $\alpha$ Syn in vitro [23, 35], CK2 rather than CK1 was demonstrated to be the major enzyme in the brain that phosphorylates S129 of human $\alpha$ Syn [13]. In a study using transgenic mice expressing human $\alpha$ Syn, S129-phosphorylated $\alpha$ Syn was shown to be preferentially colocalized with CK2 rather than CK1 a

1 MDVFMKGLSKAKEGVVAAAE

21 KTKQGVAEAAGKTKEGVLYV

41 GSKTKEGVVHGVATVAEKTK

61 EQVTNVGGAVVTGVTAVAQK

81 TVEGAGSIAAATGFVKKDQL

101 GKNEEGAPQEGILEDMPVDP

121 DNEA $\underline{\mathbf{Y}} \mathrm{EMP} \underline{\mathbf{S}} \mathrm{EEG} \underline{\mathbf{Y}} \underline{\mathrm{Y}} \underline{\mathbf{Y}} \mathrm{EPEA}$
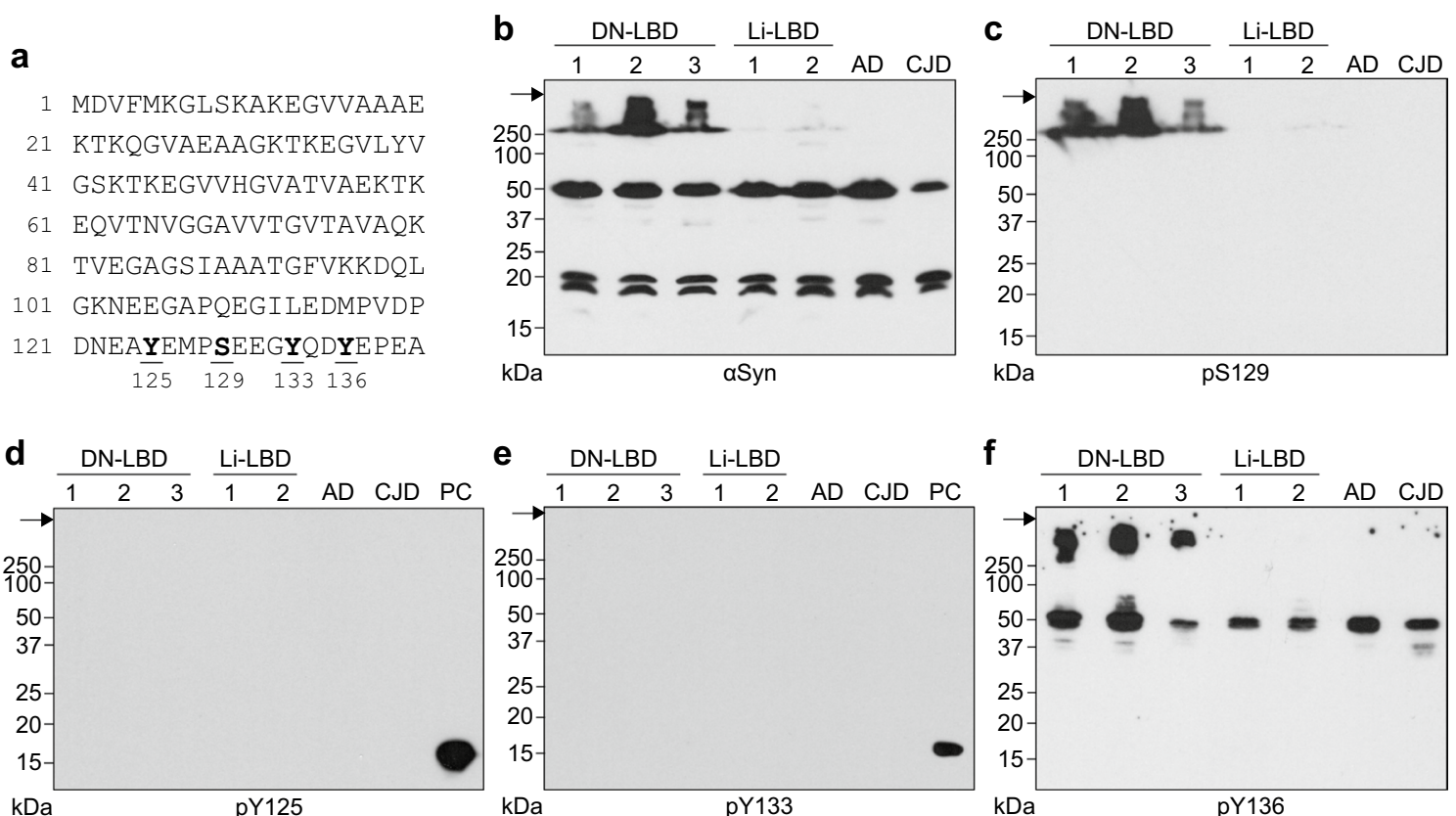

Fig. 1 Insoluble aSyn was phosphorylated at Tyr136 as well as Ser129 in the DN-LBD brain. a Amino acid sequence of human aSyn. C-terminal phosphorylation sites are shown in bold and are underlined. b-f Brain lysates from DN-LBD (cases \#1, \#2, and \#3), Li-LBD (cases \#1 and \#2), AD, and CJD patients were analyzed by SDS-PAGE followed by immunoblotting with $\mathbf{b}$ anti-aSyn antibody D119, c anti-pS129-aSyn antibody ab51253, d anti-pY125-aSyn antibody, e anti-pY133-aSyn antibody, and $\mathbf{f}$ anti-pY136-aSyn antibody. Molecular mass markers are indicated in kDa on the left side of each panel. Arrows indicate the top of the gel. In d and e, WT r-aSyn $(40 \mu \mathrm{g})$ was incubated in the presence of $100 \mathrm{ng} / \mathrm{mL} \mathrm{Syk} \mathrm{(S52-10G;}$ SignalChem Pharmaceuticals) and $200 \mu \mathrm{M}$ ATP in $100 \mu \mathrm{L}$ of reaction buffer $\left(20 \mathrm{mM}\right.$ Tris- $\mathrm{HCl}, \mathrm{pH} 7.5,50 \mathrm{mM} \mathrm{KCl}$, and $\left.10 \mathrm{mM} \mathrm{MgCl}_{2}\right)$ at $37^{\circ} \mathrm{C}$ for $1 \mathrm{~h}$, and the sample containing WT r-aSyn $(2 \mu \mathrm{g})$ was loaded as a positive control (PC) 
in the nuclei [34]. Furthermore, the catalytic $(\alpha)$ and regulatory $(\beta)$ subunits of $C K 2$ have been shown to be present in the insoluble fraction of the LBD brain [13] and in LB in the PD brain [26], respectively. In contrast, CK1 isoform delta (CK1ס) was shown to be absent in LB in the PD and LBD brain [31]. These studies suggested a role of CK2 in LB formation. CK2 has been shown to exhibit tyrosine kinase activity in cultured mammalian cells [3, 33]. In yeast, CK2 has also been reported to phosphorylate tyrosine 184 (Y184) of the nucleolar immunophilin, Fpr3, through prior phosphorylation at serine 186 (S186) at the +2 position [37], suggesting that phosphorylated serine residues play a key role in mediating phosphorylation of neighboring tyrosine residues. In the case of $\alpha$ Syn, pS129 and pY125 were shown to have no effect on each other in Drosophila by immunoblotting analysis [7] and in vitro by immunoblotting and MALDITOF mass spectrometry (MS) [11], while a study using in vitro NMR spectroscopy reported that pY125 primes efficient phosphorylation of S129 by CK1 [16]. Therefore, although tyrosines Y125, Y133, and Y136 are located in close proximity to S129 at the C-terminus of $\alpha$ Syn, it is not clear whether there is phosphorylation cross-talk between these sites, and whether such cross-talk is functionally relevant to the pathogenesis of LB disease. Here, we report that insoluble $\alpha$ Syn is highly phosphorylated at Y136 as well as S129 in the LBD brain. Furthermore, experimental manipulation of the phosphorylation state demonstrated that phosphorylation of S129 by CK2 mediates phosphorylation of Y136 and thus suppresses the formation of $\alpha$ Syn aggregates.

\section{Materials and methods Reagents}

Anti- $\alpha$ Syn polyclonal antibody (D119) and monoclonal antibody (Syn204) were obtained from Bioworld Technology Inc. and Cell Signaling Technology, respectively, and were used for western blotting. Anti-pS129- $\alpha$ Syn polyclonal antibody (D1R1R) and anti- $\beta$-actin polyclonal antibody (ab8227) were obtained from Cell Signaling Technology and Abcam, respectively, and were used for western blotting. Monoclonal antibody specific for pS129- $\alpha$ Syn (ab51253) and polyclonal antibodies against pY125- $\alpha$ Syn (ab10789), pY133- $\alpha$ Syn (ab194910), and pY136- $\alpha$ Syn (ab194775) used for western blotting and immunohistochemical staining were purchased from Abcam. Casein kinase 2 (CK2, P6010L) was obtained from New England Biolabs Inc. ATP (A2383) was acquired from Sigma-Aldrich.

\section{Brains of patients}

LBD brain tissues were obtained at autopsy from five patients with histopathologically confirmed clinical diagnosis. Three of these subjects had diffuse neocortical LBD (DN-LBD), and the remaining two cases had limbic LBD (Li-LBD) according to Braak staging. Brain tissues were obtained at autopsy from a patient with a neuropathological diagnosis of Alzheimer's disease (AD) based on the presence of neurofibrillary tangles and neuritic plaque. The brain specimens showed pure $\mathrm{AD}$ with little or no coexisting LB disease. Prion disease brain tissue specimens were obtained at autopsy from a patient with sporadic Creutzfeldt-Jakob disease (CJD), which was diagnosed as the classical MM1 subtype according to the genotype at codon 129 of the PRNP gene and the physicochemical properties of abnormal prion protein $\left(\operatorname{PrP}^{\mathrm{Sc}}\right)$. For DN-LBD cases \#1, \#2, and \#3, the individuals were 92,80 , and 91 years old at the time of death, respectively. Li-LBD cases \#1 and \#2 were 80 and 87 years old at the time of death, respectively. The patients with $\mathrm{AD}$ and CJD were 82 and 75 years old at the time of death, respectively. All tissues were taken from the frontal cortex and stored at $-80^{\circ} \mathrm{C}$.

\section{Preparation of brain lysates}

Brain tissues were lysed with Triton-deoxycholate (DOC) lysis buffer $(50 \mathrm{mM}$ Tris- $\mathrm{HCl}, \mathrm{pH} 7.5$, containing $150 \mathrm{mM} \mathrm{NaCl}, 0.5 \%$ Triton $\mathrm{X}-100,0.5 \%$ sodium deoxycholate, $2 \mathrm{mM}$ EDTA, and protease inhibitors) for $30 \mathrm{~min}$ at $4{ }^{\circ} \mathrm{C}$. After centrifugation for $2 \mathrm{~min}$ at $2000 \times g$, the supernatant was collected and stored at $-80^{\circ} \mathrm{C}$ until use. The total protein concentration of the lysates was measured using a bicinchoninic acid (BCA) protein assay kit (23227; Pierce).

\section{Recombinant human a-synuclein expression and purification}

The purification of recombinant human $\alpha$ Syn ( $r-\alpha$ Syn) and mutants was performed as described previously [29]. Briefly, plasmids carrying the DNA sequence encoding $\mathrm{N}$-terminal His-tagged human wild-type (WT) or nonphosphorylatable mutants with substitution of alanine for S129 (S129A) or Y136 (Y136A) were subcloned into the vector pET11a (69436-3; Novagen). The products encoded on the plasmid were overexpressed in competent BL21 DE3 Escherichia coli cells (DS250; BioDynamics Laboratory) at $37{ }^{\circ} \mathrm{C}$ for $16 \mathrm{~h}$ using MagicMedia $E$. coli Expression Medium (K6815; Invitrogen). The bacterial pellets were suspended in CelLytic B (B7435; SigmaAldrich) in the presence of $1 \mathrm{~g} / \mathrm{mL}$ lysozyme (120-02674; Wako) and $500 \mathrm{U} / \mathrm{mL}$ benzonase nuclease (70664-3; Novagen). The lysate was centrifuged at $10,000 \mathrm{rpm}$ for $30 \mathrm{~min}$ at $4{ }^{\circ} \mathrm{C}$, and the supernatant was incubated with Ni-NTA Superflow resin (30430; Qiagen) at room temperature for $30 \mathrm{~min}$, and then loaded onto a gravity flow column (Muromac mini-column; Muromachi Chemical 
Inc.). The His-tagged proteins were eluted with a buffer containing $300 \mathrm{mM} \mathrm{NaCl}, 50 \mathrm{mM}$ Tris- $\mathrm{HCl}$ ( $\mathrm{pH} \mathrm{8.0)}$, and $250 \mathrm{mM}$ imidazole, and dialyzed against $10 \mathrm{mM}$ phosphate buffer ( $\mathrm{pH}$ 7.0) in cellulose dialysis tubing (68035; Thermo Scientific) at $4{ }^{\circ} \mathrm{C}$ overnight. Cleavage of the His-tagged from the proteins followed by removal of uncleaved His-tagged proteins was performed using the TAGZyme system (34300; Qiagen). The non-tagged proteins were then dialyzed against deionized distilled water in cellulose dialysis tubing at $4{ }^{\circ} \mathrm{C}$ overnight and filtered with a $0.2-\mu \mathrm{m}$ syringe filter (SLLGH25; Millipore). The purity of $r-\alpha$ Syn was $\geq 99.9 \%$ as estimated by SDSPAGE and western blotting. After purification, aliquots of $r-\alpha$ Syn were stored at $-80{ }^{\circ} \mathrm{C}$ until use.

\section{In vitro phosphorylation of recombinant a-synuclein}

$\mathrm{r}-\alpha \operatorname{Syn}(40 \mu \mathrm{g})$ was incubated in the presence of 400 units of casein kinase 2 and $200 \mu \mathrm{M}$ ATP in $100 \mu \mathrm{L}$ of reac-

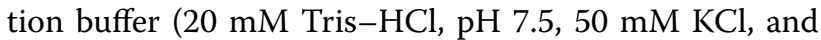
$10 \mathrm{mM} \mathrm{MgCl}_{2}$ ) at $37^{\circ} \mathrm{C}$.

\section{Gel electrophoresis and immunoblotting}

For SDS-PAGE, samples were boiled for $5 \mathrm{~min}$ at $95{ }^{\circ} \mathrm{C}$ with SDS loading buffer $(62.5 \mathrm{mM}$ Tris- $\mathrm{HCl}, \mathrm{pH} 6.8$, containing 5\% 2-mercaptoethanol, 2\% SDS, 5\% sucrose, and $0.005 \%$ bromophenol blue) and were separated by $15 \%$ SDS-PAGE. For BN-PAGE, samples were prepared in a buffer containing $0.25 \%$ Coomassie Brilliant Blue G-250 and separated by $4-16 \%$ Bis-Tris native PAGE (BN1004BOX; Invitrogen). The proteins were transferred onto Immobilon-P membranes (IPVH304F0; Millipore) in transfer buffer containing $15 \%$ methanol followed by blocking with $5 \%$ nonfat dry milk in TBST (10 mM Tris$\mathrm{HCl}, \mathrm{pH} 7.8,100 \mathrm{mM} \mathrm{NaCl}, 0.1 \%$ Tween 20) for $2 \mathrm{~h}$ at $4{ }^{\circ} \mathrm{C}$. Membranes were then probed with specific primary antibodies (1:2500) and appropriate horseradish peroxidase-conjugated secondary antibody (111-035-003 or 115-035-003, 1:5000; Jackson ImmunoResearch Labs). Immunoreactive bands were visualized using ChemiLumi One L (07880-70; Nacalai Tesque) or ECL prime Western Blotting Detection Reagents (PRN2232; GE Healthcare Life Sciences).

\section{Immunohistochemical staining}

The brain tissues were fixed in $20 \%$ neutral buffered formalin, embedded in paraffin, cut into Sects. $8 \mu \mathrm{m}$ thick with a microtome, and placed on glass slides. After deparaffinization and rehydration, the tissue sections were heated for $40 \mathrm{~min}$ at $98{ }^{\circ} \mathrm{C}$ for antigen retrieval. All sections were immersed in hydrogen peroxide $(0.3 \%)$ solution for $10 \mathrm{~min}$ to quench endogenous peroxidase activity and incubated with specific primary antibodies (1:100) diluted in PBS containing 1\% BSA for 2 h. Primary antibody binding was detected by the labeled streptavidin-biotin method (DAKO). Peroxidase-conjugated streptavidin was visualized with 3'3-diaminobenzidine (7411-49-6; Wako) as the chromogen. Immunostained sections were lightly counterstained with Mayer's hematoxylin.

\section{Gel staining and in-gel digestion}

After separation of proteins by SDS-PAGE, the gels were stained at room temperature for $2 \mathrm{~h}$ with Coomassie Brilliant Blue solution (11642; Nacalai Tesque). The stained bands near $16 \mathrm{kDa}$ were excised and soaked in $50 \mathrm{mM}$ Tris- $\mathrm{HCl}, \mathrm{pH} 8.0$, containing 50\% acetonitrile for $30 \mathrm{~min}$. The gel was dried in a Speed-Vac (Savant) and incubated in $50 \mathrm{mM}$ triethylammonium bicarbonate containing proteomics grade trypsin (T7575; Sigma-Aldrich) at $37{ }^{\circ} \mathrm{C}$ for $20 \mathrm{~h}$. The digests were extracted from the gel with $100-200 \mu \mathrm{L}$ of $0.1 \%$ TFA containing $60 \%$ acetonitrile. These extracts were evaporated in a Speed-Vac and stored at $-80{ }^{\circ} \mathrm{C}$ until assayed.

\section{Nano-flow liquid chromatography-ion trap mass spectrometry (LC-MS/MS)}

Peptides were resuspended in $0.1 \%$ formic acid containing $2 \%$ acetonitrile. Measurements were performed on a nano-flow high-performance liquid chromatography (HPLC) system (EASY-nLC 1200; Thermo Fisher Scientific). The samples were loaded onto packed nanocapillary columns $(0.075 \mathrm{~mm}$ I.D. $\times 125 \mathrm{~mm} \mathrm{~L}$, particle diameter $3 \mu \mathrm{m}$, NTCC-360/75-3-123; Nikkyo Technos Co., Ltd.), which were eluted at a flow rate of $300 \mathrm{~nL} / \mathrm{min}$ with a $2-80 \%$ linear gradient of acetonitrile for $80 \mathrm{~min}$. Eluting peptides were detected with an ion trap mass spectrometer (QExactive HF; Thermo Fisher Scientific). For ionization, the spray voltage and capillary temperature were set to $2.0 \mathrm{kV}$ and $250{ }^{\circ} \mathrm{C}$, respectively. The mass acquisition method consisted of one full MS survey scan with an Orbitrap resolution of 60,000 followed by mass spectrometry (MS/MS) of the most abundant precursor ions from the survey scan with an Orbitrap resolution of 15,000 . Dynamic exclusion for MS/MS was set to $30 \mathrm{~s}$. MS was performed with a scan range of $350-1800 \mathrm{~m} / \mathrm{z}$ in positive ion mode, followed by data-dependent MS/ MS using the HCD operating mode on the top 15 ions in order of abundance. The data were analyzed with Proteome Discoverer (Thermo Fisher Scientific) and Mascot software (Matrix Science).

\section{Transmission electron microscopy (TEM)}

Negative staining was performed on 400 mesh copper grids with a carbon support film. Aliquots of the samples were adsorbed onto the grids, and the residual solution was carefully removed from the grid surface using filter 
paper. The grids were stained with $2 \%$ uranyl acetate. Once dry, the samples were viewed with a transmission electron microscope (TEM) (JEM-2000FX; JEOL) at $200 \mathrm{kV}$.

\section{Thioflavin T (ThT) assay}

$\mathrm{r}$ - $\alpha$ Syn $(40 \mu \mathrm{g})$ was prepared in 96-well optical black-bottomed plates (265301; Nunc) in $100 \mu \mathrm{L}$ of reaction buffer (20 mM Tris-HCl, pH 7.5, $50 \mathrm{mM} \mathrm{KCl,} 10 \mathrm{mM} \mathrm{MgCl}$, and $10 \mu \mathrm{M}$ thioflavin $\mathrm{T}$ [ThT]). The 96-well plates were covered with sealing tape (236366; Nunc) and incubated at $40{ }^{\circ} \mathrm{C}$ in a plate reader (FLUOstar Omega plate reader; BMG Labtech) with intermittent shaking, consisting of $30 \mathrm{~s}$ of double orbital shaking at $500 \mathrm{rpm}$ and no shaking for $30 \mathrm{~s}$. ThT fluorescence intensity on the bottom of the plates was measured every $10 \mathrm{~min}$ to monitor the kinetics of amyloid fibril formation using monochromators with excitation and emission wavelengths of $450 \pm 10$ and $480 \pm 10 \mathrm{~nm}$, respectively. Lag phase was defined as the time required to reach fluorescence greater than or equal to the mean fluorescence intensity for all samples within the first $24 \mathrm{~h}$ of reaction plus $4 \times$ the standard deviation.

\section{Preparation of r-aSyn seeds}

$\mathrm{r}-\alpha$ Syn $(40 \mu \mathrm{g})$ was incubated in $20 \mathrm{mM}$ Tris- $\mathrm{HCl}, \mathrm{pH}$ 7.5 , containing $50 \mathrm{mM} \mathrm{KCl}$, and $10 \mathrm{mM} \mathrm{MgCl}_{2}$, at $37^{\circ} \mathrm{C}$ under agitation at $2000 \mathrm{rpm}$ for 3 days. As a control for agitated $r-\alpha$ Syn, the mix was prepared without incubation and agitation immediately before use. The morphology of $r-\alpha$ Syn seeds was confirmed by TEM.

\section{Cell culture, treatment of r-aSyn seeds, and transfection of plasmids}

Human neuroblastoma SH-SY5Y cells were maintained at $37{ }^{\circ} \mathrm{C}$ in $5 \% \mathrm{CO}_{2}$ in DMEM/Ham's F12 medium (042-30795; Wako) containing 15\% fetal bovine serum (SH30910; GE Healthcare Hyclone), penicillin-streptomycin (168-23191; Wako), and MEM Non-essential Amino Acids Solution (139-15651; Wako). Introduction of $r-\alpha$ Syn seeds and/or pcDNA3.1 plasmid encoding human WT or Y136A mutant $\alpha$ Syn into cells was performed using Lipofectamine ${ }^{\mathrm{TM}}$ LTX (Invitrogen) according to the manufacturer's instructions. Lipofectamine/r- $\alpha$ Syn seeds and/or plasmid complexes were prepared in Optimem (31985062; Gibco) by mixing Lipofectamine LTX reagent in the presence or absence of $\mathrm{r}$ - $\alpha$ Syn seeds $(4 \mu \mathrm{g})$ or plasmid $(5 \mu \mathrm{g})$. Cells were cultured to $40-50 \%$ confluence in 6 -well plates and treated with the complexes. For CK2 inhibitor treatment, cells were incubated with 10 or $100 \mathrm{nM}$ 4,5,6,7-tetrabromobenzotriazole (TBB, ab120988; Abcam) for $1 \mathrm{~h}$ before addition of the complexes. cells were incubated for $3 \mathrm{~d}$ after introduction of $r-\alpha$ Syn seeds and/or plasmid.

\section{Preparation of lysate from cell culture}

Cells were washed with PBS and harvested, and cellular proteins were extracted with Triton-DOC lysis buffer. The total protein concentration of the lysates was measured using a BCA protein assay kit (23227; Pierce).

\section{Statistics}

Statistical analyses were performed with OriginPro 2015 (OriginLab). The 2-tailed Student's $t$ test was used for comparisons between two groups. One-way analysis of variance (ANOVA) followed by the Tukey-Kramer test was used for comparisons among more than two groups. In all analyses, $P<0.05$ was taken to indicate statistical significance.

\section{Results}

Insoluble aggregates of aSyn are predominantly

phosphorylated on $\mathrm{Y} 136$ as well as $\mathrm{S} 129$

within the C-terminal region in the LBD brain

Brain lysates from LBD patients were analyzed by SDSPAGE followed by immunoblotting with anti- $\alpha$ Syn antibody, and all exhibited a band at approximately $20 \mathrm{kDa}$ with another band just below $20 \mathrm{kDa}$ likely corresponding to full-length and cleaved $\alpha$ Syn, respectively (Fig. 1b). All lysates also exhibited an $\alpha$ Syn-positive band at approximately $50 \mathrm{kDa}$, which was presumably due to the oligomeric and/or ubiquitinated $\alpha$ Syn. Although these $\alpha$ Syn-positive bands did not differ significantly between cases, multimeric $\alpha$ Syn with molecular weight $>250 \mathrm{kDa}$ was observed only in three cases of DN-LBD (Fig. 1b, Additional file 1: Fig. S1). Immunostaining with an antibody against pS129- $\alpha$ Syn detected only a major band at $>250 \mathrm{kDa}$ in all cases of DN-LBD (Fig. 1c, Additional file 1: Fig. S1). A band of $>250 \mathrm{kDa}$ was detected with antibodies against $\alpha$ Syn and pS129- $\alpha$ Syn in Li-LBD case $\# 2$, but not case \#1, but the intensity was significantly lower than in DN-LBD (Fig. 1b, c), indicating that multimer formation and pS129 of $\alpha$ Syn are relevant to disease progression. Consistent with our previous report [29], these results suggest that the detergent-insoluble multimer of $\alpha$ Syn highly phosphorylated at S129 with molecular weight $>250 \mathrm{kDa}$ is present in the DN-LBD brain. We next examined the presence of $\alpha$ Syn phosphorylated at C-terminal tyrosine residues surrounding S129. No signals were detected with antibodies against pY125$\alpha$ Syn and pY133- $\alpha$ Syn in any cases (Fig. 1d, e). A 50-kDa band was detected with an antibody against pY136- $\alpha$ Syn in all cases (Fig. 1f, Additional file 1: Fig. S1). Moreover, the three cases of DN-LBD showed a strong immunoreactive band with mass $>250 \mathrm{kDa}$ (Fig. 1f, Additional file 1: Fig. S1). These results suggest that the insoluble aggregates of $\alpha$ Syn are predominantly phosphorylated on Y136 
as well as S129 within the C-terminal region in the LBD brain.

We next examined phosphorylation of the C-terminus of $\alpha$ Syn in sections of the frontal cortex of DN-LBD and Li-LBD brains by immunohistochemical analysis.
Consistent with our previous study [29], the deposits showed positive staining for $\mathrm{pS} 129-\alpha$ Syn, and were also positive for pY136- $\alpha$ Syn in both DN-LBD and Li-LBD brains (Fig. 2a). The numbers of pS129- $\alpha$ Syn and pY136$\alpha$ Syn deposits were significantly greater in DN-LBD than a

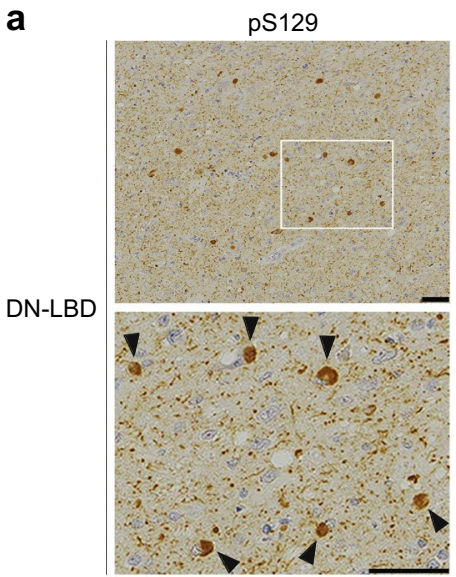

pS129

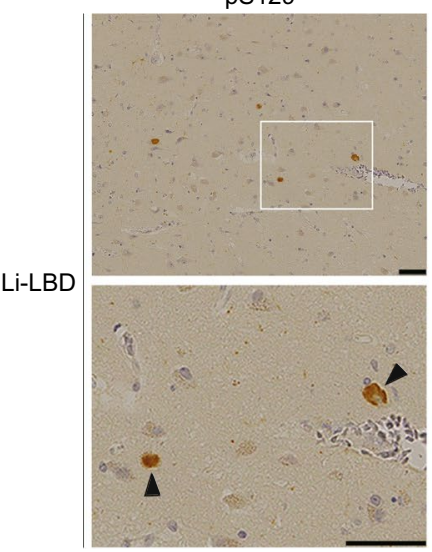

b

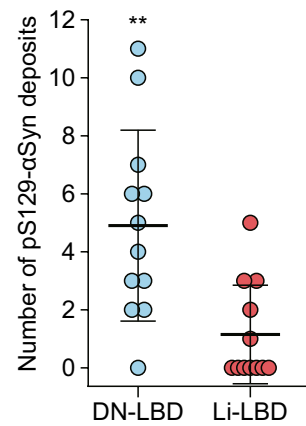

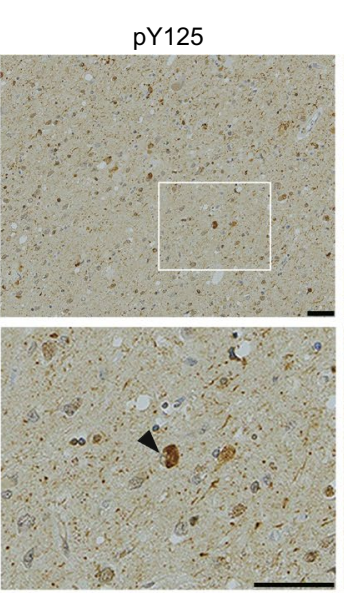

pY125
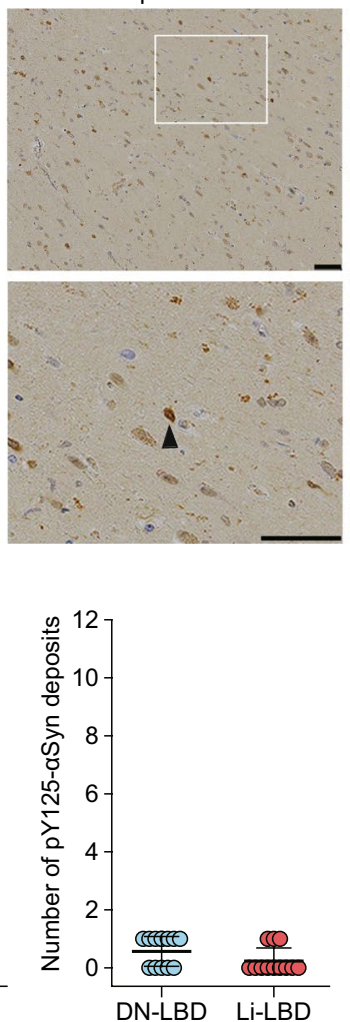

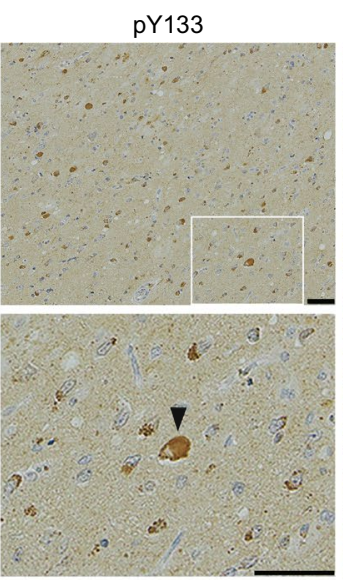

pY133
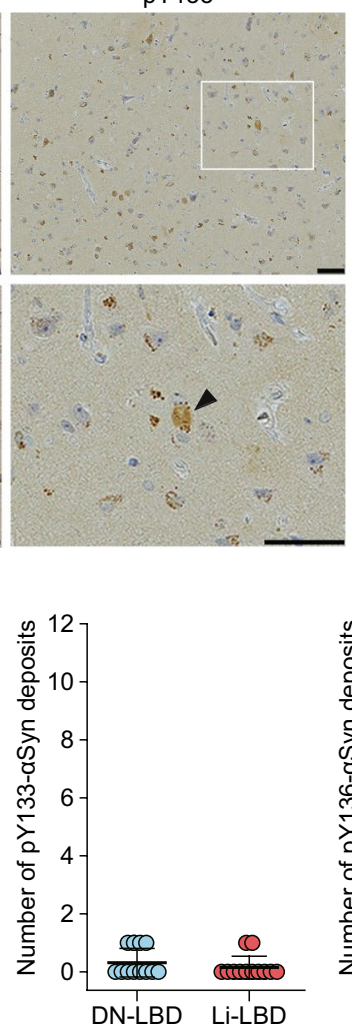

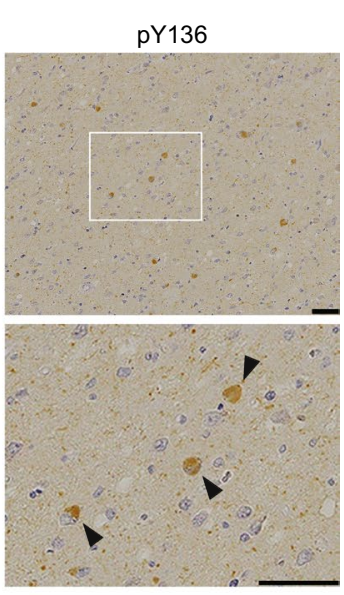

pY136
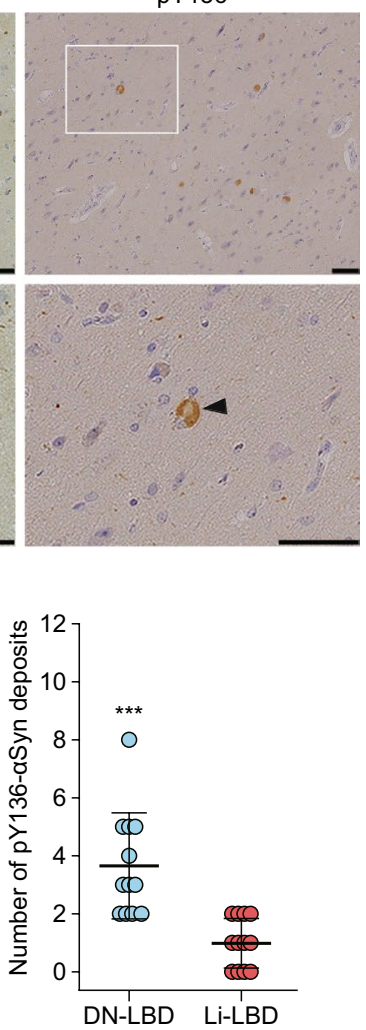

Fig. 2 Deposits of phosphorylated aSyn at C-terminal tyrosine residues are present in LBD brain. a Immunohistochemical staining using antibodies against pS129-aSyn ab51253, pY125-aSyn, pY133-aSyn, and pY136-aSyn in the frontal cortex from two patients with DN-LBD (case \#3) and Li-LBD (case \#2). Regions surrounded by white rectangles in the upper panels are magnified and shown in the lower panels. Typical pathological aSyn deposits are indicated by arrowheads. Scale bars, $50 \mu \mathrm{m}$. b The number of phosphorylated aSyn deposits $>2 \mu \mathrm{m}^{2}$ was counted in 12 randomly selected areas of $10 \mathrm{~mm}^{2}$ in each tissue specimen using ImageJ. Data are presented as means \pm standard deviation. Statistical significance was determined using the 2 -tailed Student's $t$ test. ${ }^{* *} s<0.01$, ${ }^{* * *} P<0.001$ vs. Li-LBD 
Li-LBD (Fig. 2b). Deposits were not detected in the brain of a non-DLB patient by immunohistochemical analysis using anti-pS129- $\alpha$ Syn antibody (Additional file 1: Fig. S2). Both DN-LBD and Li-LBD brains showed positive staining with anti-pY125- $\alpha$ Syn and anti-pY133- $\alpha$ Syn antibodies (Fig. 2a), but pT125- $\alpha$ Syn and pY133- $\alpha$ Syn deposits were present at low levels in both DN-LBD and Li-LBD compared to pS129- $\alpha$ Syn and pY136- $\alpha$ Syn deposits in DN-LBD (Fig. 2b). The lack of detectable pY125 and pY133 in the brain by immunoblotting was likely due to low levels of the deposits, and phosphorylated $\alpha$ Syn may not be detected by immunoblotting in brains with less than two phosphorylated deposits per $10 \mathrm{~mm}^{2}$ of tissue. There were no significant differences in the numbers of pY125- $\alpha$ Syn and pY133- $\alpha$ Syn deposits between the two types of LBD (Fig. 2b). Thus, the results of immunohistochemical analysis indicated the presence of pS129- $\alpha$ Synand pY136- $\alpha$ Syn-positive deposits in larger amounts in the brains of patients with DN-LBD than Li-LBD, and that pY125- $\alpha$ Syn and pY133- $\alpha$ Syn are present in relatively small amounts in the brains of patients with both types of LBD.

\section{Ser129 phosphorylation by CK2 promotes the formation of insoluble aSyn aggregates in vitro}

Analysis of WT $r-\alpha$ Syn by SDS-PAGE followed by immunoblotting with anti- $\alpha$ Syn antibody revealed detergent-insoluble aggregates $>250 \mathrm{kDa}$ after 15 days of incubation, which were formed more efficiently in the presence of both CK2 and ATP than in the absence of either (Additional file 1: Fig. S3). Insoluble aggregates and $16-\mathrm{kDa}$ WT $\mathrm{r}-\alpha$ Syn monomer incubated with CK2 and ATP were detected by anti-pS129- $\alpha$ Syn antibody, whereas no immunoreactivity was detected with antipS129- $\alpha$ Syn antibody for WT $r-\alpha$ Syn incubated under other conditions (Additional file 1: Fig. S3). The insoluble aggregates of S129-phosphorylated WT r- $\alpha$ Syn were detected after 1 day of incubation (Fig. 3a, b). Meanwhile, S129A r- $\alpha$ Syn incubated with CK2 and ATP was not phosphorylated at Ser129 and formed no insoluble aggregates (Fig. 3a, b). TEM revealed that WT $r-\alpha$ Syn subjected to 15-day incubation with CK2 and ATP consisted exclusively of amorphous aggregates, but no such aggregates were seen without incubation (Fig. 3c). The amorphous aggregates were present in relatively small amounts in WT $r$ - $\alpha$ Syn incubated in the absence of CK2 and ATP (Additional file 1: Fig. S4). These results were consistent with a previous report [29] indicating that CK2-induced pS129 accelerated aggregate formation of WT $r-\alpha S y n$, and the insoluble aggregates were observed in the mass range $>250 \mathrm{kDa}$ as in the $\mathrm{DN}-\mathrm{LBD}$ brain. Blue native (BN)-PAGE followed by immunoblotting analysis with anti- $\alpha$ Syn antibody of WT $r-\alpha$ Syn incubated for
15 days revealed the formation of $\alpha$ Syn aggregates with a molecular weight of $\sim 1048 \mathrm{kDa}$ in addition to a band of monomeric $\alpha$ Syn at approximately $60 \mathrm{kDa}$ (Fig. 3d). The aggregated WT r- $\alpha$ Syn was also formed more efficiently in the presence of both CK2 and ATP than in the absence of either, suggesting that pS129 accelerates aggregate formation of $\alpha$ Syn (Fig. 3d, e).

\section{CK2 phosphorylates Y125 and Y136 of aggregated aSyn through prior S129 phosphorylation in vitro}

WT $r-\alpha$ Syn or S129A $r-\alpha$ Syn was incubated with CK2 and ATP for 7 days, separated by SDS-PAGE, and the gel portion corresponding to a molecular weight of $\sim 16 \mathrm{kDa}$ was cut out as shown in Additional file 1: Fig. S5. The gel slice was digested with trypsin and analyzed by LC-MS/ MS. As shown in Table 1, we identified 23 and seven phosphorylated peptides from WT and S129A r- $\alpha$ Syn, respectively. LC-MS/MS analysis detected pS129 in WT r- $\alpha$ Syn. Both WT $r-\alpha S y n$ and S129A r- $\alpha$ Syn contained pS87. WT $r-\alpha$ Syn showed phosphorylation at four threonine residues, i.e., T44, T54, T59, and T64, while S129A r- $\alpha$ Syn showed phosphorylation at five threonine residues, i.e., T22, T44, T54, T59, and T75. There have been no previous reports of phosphorylation of $\alpha$ Syn at T22, T44, T54, T59, T64, T75, and S87 by CK2, and the number of peptides including these residues with phosphorylation was very low in comparison to peptides with pS129 (Table 1). Therefore, CK2 may phosphorylate $r-\alpha$ Syn on these residues at very low levels in vitro. Moreover, peptides with pY125 and pY136 were identified in WT r- $\alpha$ Syn. The number of peptides with pY136 was comparable to that of peptides with pS129, and only one peptide with pY125 was detected. In contrast, no peptides with $\mathrm{C}$-terminal tyrosine phosphorylation were identified in S129A r- $\alpha$ Syn. After SDS-PAGE of WT $r-\alpha$ Syn, immunoblotting with anti-pY125- $\alpha$ Syn antibody and anti-pY136- $\alpha$ Syn antibody detected detergent-insoluble aggregates $>250 \mathrm{kDa}$ after 7 and 3 days of incubation with CK2 and ATP, respectively (Fig. 4a, b). No immunoreactivity was detected on blots of S129A with anti-pY125- $\alpha$ Syn or anti-pY136- $\alpha$ Syn antibody (Fig. 4a, b). In BN-PAGE followed by immunoblotting analysis of WT $r$ - $\alpha$ Syn incubated for 15 days, bands of approximately $1048 \mathrm{kDa}$ were detected with antibodies against pY125- $\alpha$ Syn and pY136- $\alpha$ Syn only in the presence of CK2 and ATP (Fig. 4c, d). Moreover, pS129 and pY136 were abolished and the formation of insoluble aggregates was significantly inhibited by the addition of alkaline phosphatase in WT $r-\alpha$ Syn incubated with CK2 and ATP (Additional file 1: Fig. S6). These results suggested that CK2 phosphorylates Y125 and Y136 of aggregated WT 

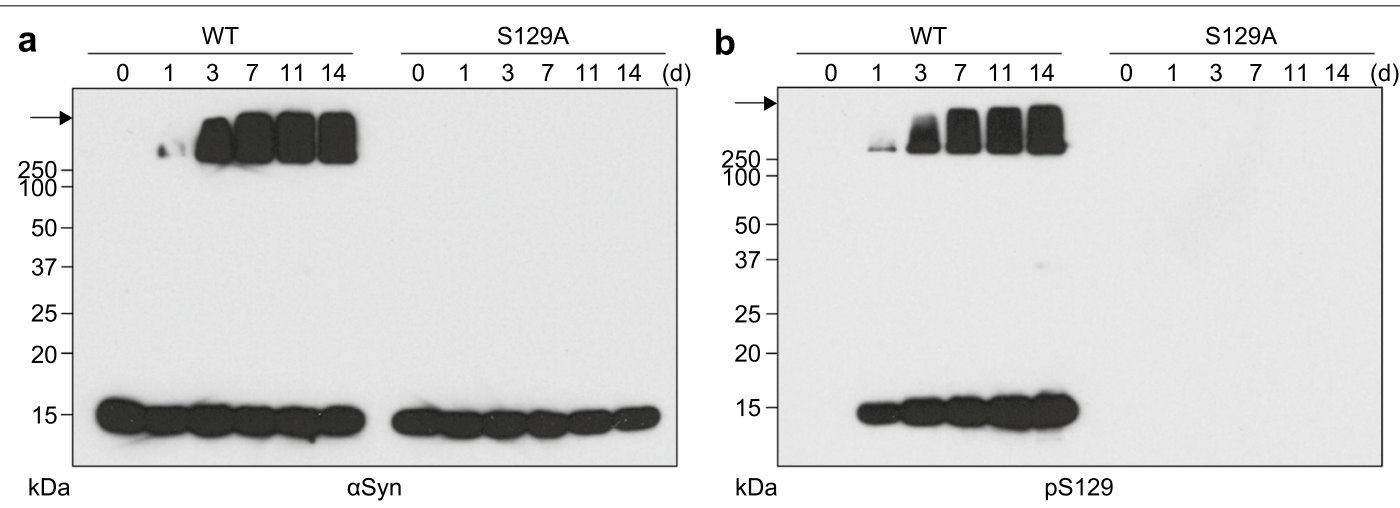

C
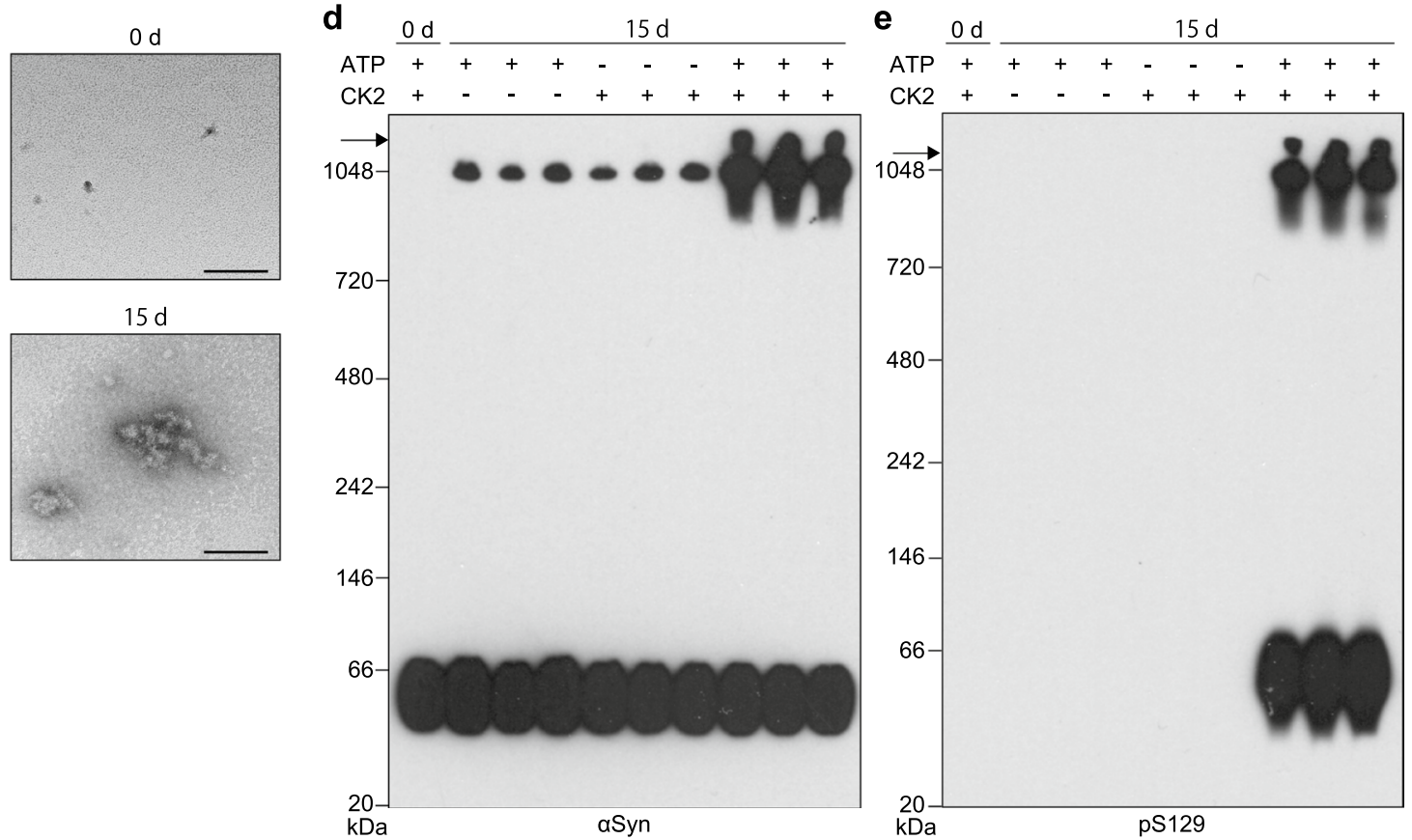

Fig. 3 Ser129 phosphorylation by CK2 accelerates aSyn aggregate formation. a, b WT r-aSyn or S129A r-aSyn after 0-14 days of incubation with CK2 and ATP was analyzed by SDS-PAGE followed by immunoblotting with $\mathbf{a}$ anti-aSyn antibody D1 19 and $\mathbf{b}$ anti-pS129-aSyn antibody ab51253. cWT r-aSyn after 0 or 15 days of incubation with CK2 and ATP was examined by TEM. Bars, $100 \mathrm{~nm}$. d, e WT r-aSyn after 0 or 15 days of incubation in the presence $(+)$ or absence $(-)$ of CK2 or ATP was analyzed by BN-PAGE followed by immunoblotting with $\mathbf{d}$ anti-aSyn antibody D1 19 and $\mathbf{e}$ anti-pS129-aSyn antibody ab51253. Molecular mass markers are indicated in kDa on the left side of each panel. Arrows indicate the top of the gel. In $\mathbf{a}, \mathbf{b}, \mathbf{d}$ and $\mathbf{e}$, one representative blot from three independent experiments is shown

$\mathrm{r}-\alpha$ Syn in vitro, and that the tyrosine phosphorylation is mediated through pS129.

\section{Y136 phosphorylation prevents S129 phosphorylation and aggregate formation of aSyn in vitro}

On SDS-PAGE followed by immunoblotting, Y136A $r-\alpha$ Syn incubated for 14 days also exhibited detergentinsoluble aggregates $>250 \mathrm{kDa}$, which were most strongly detected in the presence of both CK2 and ATP (Additional file 1: Fig. S7). Insoluble aggregates $>250 \mathrm{kDa}$ as well as $16 \mathrm{kDa}$ monomer of Y136A r- $\alpha$ Syn incubated with
CK2 and ATP were detected by anti-pS129- $\alpha$ Syn antibody (Additional file 1: Fig. S7), while no immunoreactivity was detected with anti-pY136- $\alpha$ Syn antibody in any samples (Additional file 1: Fig. S7). Although no $\alpha$ Synpositive band $>250 \mathrm{kDa}$ of WT $\mathrm{r}-\alpha$ Syn or Y136A $r-\alpha$ Syn was observed within $40 \mathrm{~h}$ of incubation in the absence of CK2 and ATP (Additional file 1: Fig. S8), the band was detected after $8 \mathrm{~h}$ of incubation in the presence of CK2 and ATP (Fig. 5a). The intensity of the $\alpha$ Syn-positive band $>250 \mathrm{kDa}$ of Y136A $\mathrm{r}-\alpha$ Syn was significantly greater than that of WT r- $\alpha$ Syn after $8 \mathrm{~h}$ of incubation 
Table 1 List of identified peptides derived from r-aSyn incubated with CK2 and ATP for 7 days

\begin{tabular}{|c|c|c|c|c|c|c|c|c|c|}
\hline Query & Start & End & Observed & $\operatorname{Mr}(\operatorname{expt})$ & $\operatorname{Mr}($ calc) & ppm & Score & Expect & Peptide \\
\hline \multicolumn{10}{|l|}{ WT } \\
\hline 5284 & 44 & 58 & 802.9034 & 1603.792 & 1603.797 & -2.97 & 65 & $3.5 \mathrm{E}-07$ & K.TpKEGVVHGVATVAEK.T \\
\hline 11012 & 46 & 80 & 1172.286 & 3513.836 & 3513.808 & 8.02 & 51 & $8.4 \mathrm{E}-06$ & K.EGVHHGVATpVAEKTKEQVTN*VGGAVVTGVTAVAQK.T \\
\hline 8325 & 59 & 80 & 746.3851 & 2236.134 & 2236.147 & -5.77 & 47 & 0.000021 & K.TKEQVTpNVGGAVVTGVTAVAQK.T \\
\hline 8336 & 59 & 80 & 1120.068 & 2238.122 & 2238.115 & 3.44 & 21 & 0.0083 & K.TpKEQ*VTN*VGGAVVTGVTAVAQK.T \\
\hline 11170 & 59 & 96 & 924.9822 & 3695.9 & 3695.914 & -3.77 & 76 & $2.4 \mathrm{E}-08$ & K.TpKEQVTNVGGAVVTGVTAVAQKTVEGAGSIAAATGFVK.K \\
\hline 5009 & 81 & 96 & 779.8777 & 1557.741 & 1557.744 & -1.95 & 88 & $1.6 \mathrm{E}-09$ & K.TVEGAGSpIAAATGFVK.K \\
\hline 12198 & 98 & 140 & 1228.245 & 4908.951 & 4908.947 & 0.96 & 20 & 0.011 & K.DQLGKN*EEGAPQEGILEDMPVDPDN*EAYEMPSpEEGYQDYEPEA \\
\hline 12214 & 98 & 140 & 1232.002 & 4923.98 & 4923.958 & 4.6 & 34 & 0.00044 & K.DQLGKN*EEGAPQEGILEDMoPVDPDNEAYEMPSEEGYQDYpEPEA \\
\hline 12225 & 98 & 140 & 1235.754 & 4938.985 & 4938.969 & 3.36 & 21 & 0.01 & K.DQLGKNEEGAPQEGILEDMoPVDPDNEAYEMoPSEEGYQDYpEPEA \\
\hline 12226 & 98 & 140 & 1235.756 & 4938.994 & 4938.969 & 5.24 & 30 & 0.0011 & K.DQLGKNEEGAPQEGILEDMoPVDPDNEAYpEMoPSEEGYQDYEPEA \\
\hline 12228 & 98 & 140 & 1236.002 & 4939.979 & 4939.953 & 5.31 & 23 & 0.0073 & K.DQLGKNEEGAPQEGILEDMoPVDPDN*EAYEMoPSEEGYQDYpEPEA \\
\hline 12229 & 98 & 140 & 1236.003 & 4939.982 & 4939.953 & 5.91 & 26 & 0.0032 & K.DQ*LGKNEEGAPQEGILEDMoPVDPDNEAYEMoPSEEGYQDYpEPEA \\
\hline 11769 & 103 & 140 & 1092.433 & 4365.703 & 4365.693 & 2.34 & 21 & 0.0077 & K.NEEGAPQEGILEDMPVDPDNEAYEMPSEEGYQDYPEPEA \\
\hline 11770 & 103 & 140 & 1456.242 & 4365.704 & 4365.693 & 2.61 & 18 & 0.017 & K.NEEGAPQEGILEDMPVDPDNEAYEMPSPEEGYQDYEPEA \\
\hline 11805 & 103 & 140 & 1461.57 & 4381.688 & 4381.688 & 0.11 & 24 & 0.004 & K.NEEGAPQEGILEDMPVDPDNEAYEMoPSpEEGYQDYEPEA \\
\hline 11806 & 103 & 140 & 1461.572 & 4381.693 & 4381.688 & 1.19 & 22 & 0.0069 & K.NEEGAPQEGILEDMPVDPDNEAYEMoPSpEEGYQDYEPEA \\
\hline 11807 & 103 & 140 & 1461.572 & 4381.694 & 4381.688 & 1.45 & 28 & 0.0015 & K.NEEGAPQEGILEDMoPVDPDNEAYEMPSpEEGYQDYEPEA \\
\hline 11808 & 103 & 140 & 1096.431 & 4381.696 & 4381.688 & 1.93 & 25 & 0.0034 & K.NEEGAPQEGILEDMPVDPDNEAYEMoPSpEEGYQDYEPEA \\
\hline 11809 & 103 & 140 & 1461.576 & 4381.707 & 4381.688 & 4.54 & 28 & 0.0017 & K.NEEGAPQEGILEDMPVDPDNEAYEMoPSpEEGYQDYEPEA \\
\hline 11851 & 103 & 140 & 1100.425 & 4397.671 & 4397.682 & -2.69 & 31 & 0.00073 & K.NEEGAPQEGILEDMoPVDPDNEAYEMoPSpEEGYQDYEPEA \\
\hline 11855 & 103 & 140 & 1466.906 & 4397.696 & 4397.682 & 3.13 & 31 & 0.00086 & K.NEEGAPQEGILEDMoPVDPDNEAYEMoPSEEGYQDYpEPEA \\
\hline 11856 & 103 & 140 & 1466.908 & 4397.701 & 4397.682 & 4.29 & 24 & 0.004 & K.NEEGAPQEGILEDMoPVDPDNEAYEMoPSEEGYQDYpEPEA \\
\hline 11870 & 103 & 140 & 1467.57 & 4399.687 & 4399.651 & 8.37 & 24 & 0.0039 & K.NEEGAPQEGILEDMoPVDPDNEAYEMoPSpEEGYQDYEPEA \\
\hline \multicolumn{10}{|l|}{ S129A } \\
\hline 3243 & 11 & 23 & 691.3577 & 1380.701 & 1380.701 & -0.32 & 28 & 0.0014 & K.AKEGVVAAAEKTpK.Q \\
\hline 1528 & 22 & 32 & 570.2711 & 1138.528 & 1138.538 & -9.45 & 46 & 0.000028 & K.TpKQGVAEAAGK.T \\
\hline 4891 & 44 & 58 & 802.8981 & 1603.782 & 1603.797 & -9.67 & 60 & $9.1 \mathrm{E}-07$ & K.TpKEGVVHGVATVAEK.T \\
\hline 3201 & 46 & 58 & 688.3341 & 1374.654 & 1374.654 & -0.57 & 42 & 0.00006 & K.EGVVHGVATPVAEK.T \\
\hline 8000 & 59 & 80 & 1119.079 & 2236.144 & 2236.147 & -1.15 & 64 & $4.2 \mathrm{E}-07$ & K.TpKEQVTNVGGAVVTGVTAVAQK.T \\
\hline 8001 & 59 & 80 & 746.3887 & 2236.144 & 2236.147 & -1.02 & 25 & 0.003 & K.TKEQVTNVGGAVVTGVTpAVAQK.T \\
\hline 5372 & 81 & 97 & 843.9226 & 1685.831 & 1685.839 & -4.98 & 52 & $6.4 \mathrm{E}-06$ & K.TVEGAGSPIAAATGFVKK.D \\
\hline
\end{tabular}

$\mathrm{p}$ indicates phosphorylation sites; o indicates oxidation site

*Indicates deamidation site

(Fig. 5b). Moreover, ThT spectroscopic assay showed an increase in fluorescence in reactions with Y136A $r-\alpha$ Syn in the presence of CK2 and ATP, but not in their absence, whereas no increase in fluorescence was observed with WT $r-\alpha$ Syn regardless of the presence or absence of CK2 and ATP within 7 days (Fig. 5c, Additional file 1: Fig. S9). Y136A $r-\alpha$ Syn amyloid fibrils were observed in reactions in the presence of CK2 and ATP on day 7 of incubation by TEM analysis (Fig. 5d, Additional file 1: Fig. S10). In contrast, amorphous aggregates, but not fibrils, were exclusively observed in reactions with WT $r-\alpha$ Syn in the presence of CK2 and ATP (Fig. 5d, Additional file 1: Fig. S10). The maximal fluorescence intensity was significantly higher in reactions with Y136A $r-\alpha$ Syn in the presence of CK2 and ATP than other reactions (Fig. 5e). The lag phase was significantly shorter in reactions with Y136A $r-\alpha$ Syn in the presence of CK2 and ATP than other reactions (Fig. 5f). These results suggest that blocking Y136 phosphorylation facilitates aggregation and amyloid fibril formation of $\alpha$ Syn. The insoluble aggregates $>250 \mathrm{kDa}$ were detected in WT $\mathrm{r}-\alpha \mathrm{Syn}$, but not Y136A $r-\alpha$ Syn, with an antibody against pY136- $\alpha$ Syn and in both WT and Y136A $r-\alpha$ Syn with an antibody against pS129- $\alpha$ Syn after $8 \mathrm{~h}$ of incubation in the presence of CK2 and ATP (Fig. 6a, b). Interestingly, the intensity of the pS129- $\alpha$ Syn-positive band of Y136A $r-\alpha$ Syn was significantly higher than that of WT r- $\alpha$ Syn at $8 \mathrm{~h}$ of incubation (Fig. 6c). Unlike WT $r-\alpha$ Syn, immunoreactivity 


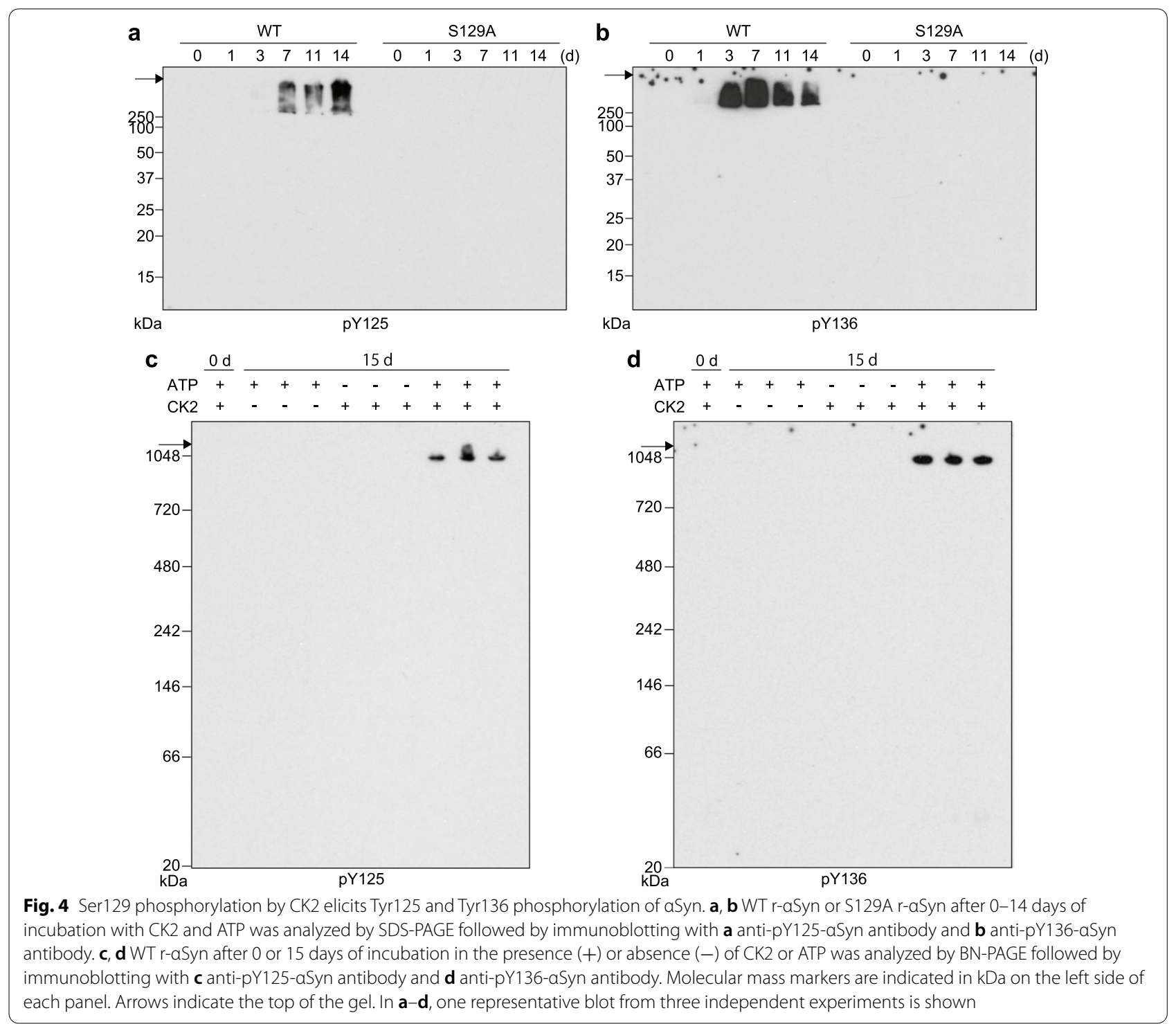

with anti-pS129- $\alpha$ Syn antibody was more prominent in the insoluble aggregates with molecular weight $>250 \mathrm{kDa}$ than $16-\mathrm{kDa}$ monomer of Y136A $\mathrm{r}-\alpha$ Syn at $8-40 \mathrm{~h}$ of incubation (Fig. 6b). The insoluble aggregates $>250 \mathrm{kDa}$ were detected at relatively low levels in WT $\mathrm{r}-\alpha$ Syn and Y136A $r-\alpha$ Syn with anti-pY125- $\alpha$ Syn antibody after $8 \mathrm{~h}$ of incubation in the presence of CK2 and ATP (Fig. 6d). The intensity of the pY125- $\alpha$ Syn-positive band of Y136A $r-\alpha$ Syn was significantly higher than that of WT $r-\alpha$ Syn at $8 \mathrm{~h}$ of incubation (Fig. 6e). Only a low level of insoluble aggregates $>250 \mathrm{kDa}$ was detected in Y136A r- $\alpha$ Syn by anti-pY133- $\alpha$ Syn antibody after 32 and $40 \mathrm{~h}$ of incubation in the presence of CK2 and ATP, whereas no immunoreactivity was detected with anti-pY133- $\alpha$ Syn antibody for WT $r-\alpha$ Syn (Additional file 1: Fig. S11).
These observations indicated that blocking of Y136 phosphorylation facilitated aggregate formation and phosphorylation at other C-terminal residues, especially S129, of $\mathrm{r}-\alpha$ Syn. The results suggest that pY136 inhibits pS129 of $\alpha$ Syn and thereby prevents aggregate formation.

\section{Exogenous oligomeric aSyn converts endogenous aSyn into insoluble aggregates with pS129 and pY136 in a prion-like manner and $Y 136$ phosphorylation is involved in protecting against $\mathrm{S} 129$ phosphorylation and aggregate formation of aSyn in cultured cells}

We reported previously that oligomeric $r-\alpha$ Syn produced by agitation shows potent prion-like seeding activity in vitro by real-time quaking-induced conversion (RTQUIC) seeding assay [29]. We confirmed the contribution 


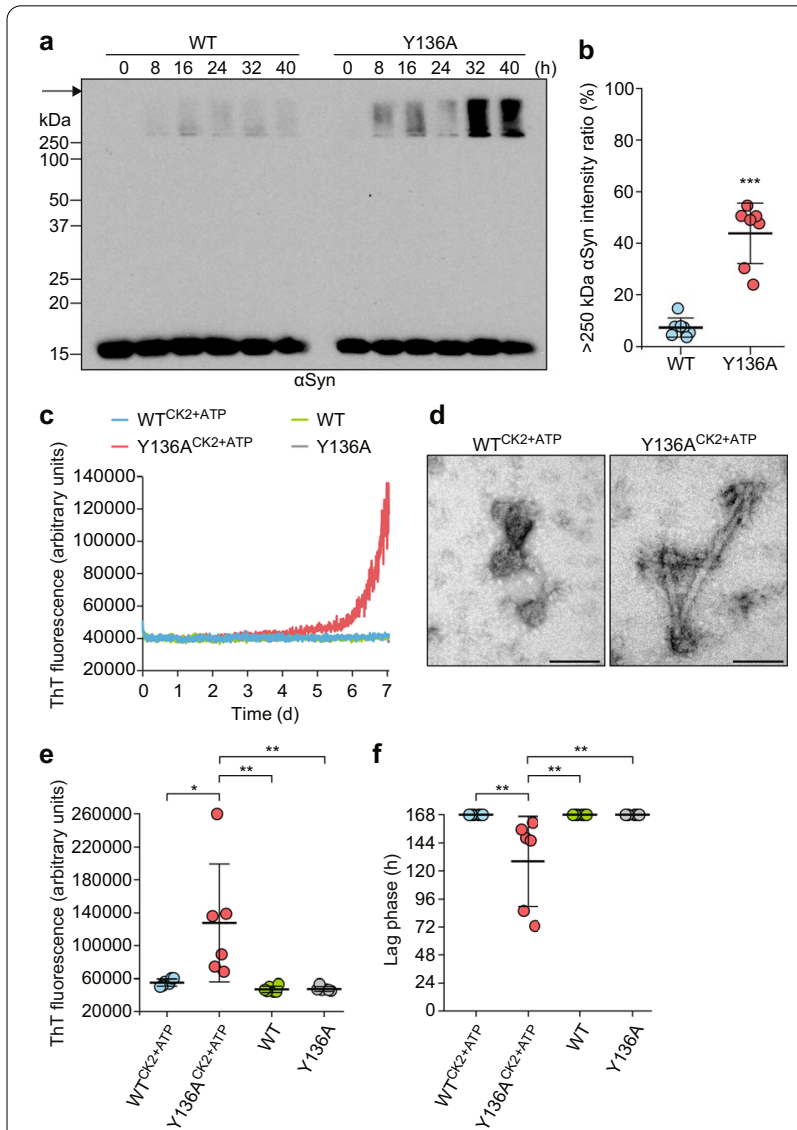

Fig. 5 Blocking Tyr136 phosphorylation promotes aggregation and amyloid fibril formation of aSyn. a WT r-aSyn or Y136A r-aSyn after $0-40 \mathrm{~h}$ of incubation with CK2 and ATP was analyzed by SDS-PAGE followed by immunoblotting with anti-aSyn antibody D1 19. Molecular mass markers are indicated in $\mathrm{kDa}$ on the left side of each panel. Arrows indicate the top of the gel. $\mathbf{b}$ Intensity ratios (\%) of immunoreactive $>250-k D a$ aSyn after $8 \mathrm{~h}$ of incubation were quantified in seven independent experiments. Data are presented as means \pm standard deviation. Statistical significance was determined using the 2 -tailed Student's $t$ test. ${ }^{* *} P<0.001$ vs. WT r-aSyn. c ThT assays were performed in reactions with WT r-aSyn in the presence (WT ${ }^{\text {CK2 }+A T P}$ ) or absence (WT) of CK2 and ATP or in reactions with Y136A r-aSyn in the presence (Y136A ${ }^{\text {CK2+ATP }}$ ) or absence (Y136A) of CK2 and ATP. The results show the kinetics of ThT fluorescence from one representative of six replicate wells for each condition. $\mathbf{d}$ The end products from reactions with WT ${ }^{\text {CK2 }}{ }^{\text {ATP }}$ or $\mathrm{Y} 136 \mathrm{~A}^{\text {CK2 }+ \text { ATP }}$ were examined by TEM. Bars, $100 \mathrm{~nm}$. Values of e maximal fluorescence intensities and $\mathbf{f}$ lag phase obtained in six individual wells in ThT assay are plotted. Data are presented as means \pm standard deviation. Statistical significance was determined using one-way ANOVA followed by Tukey-Kramer test. ${ }^{*} P<0.05,{ }^{*} P<0.01$

of phosphorylation at S129 and Y136 to aggregate formation of $\alpha$ Syn in SH-SY5Y cells using oligomeric $r-\alpha$ Syn as seeds for prion-like propagation. WT $r-\alpha$ Syn and Y136A $r-\alpha$ Syn were converted into oligomeric forms by agitation (Fig. 7a). There were no significant differences in both forms. SDS-PAGE followed by immunoblotting analysis

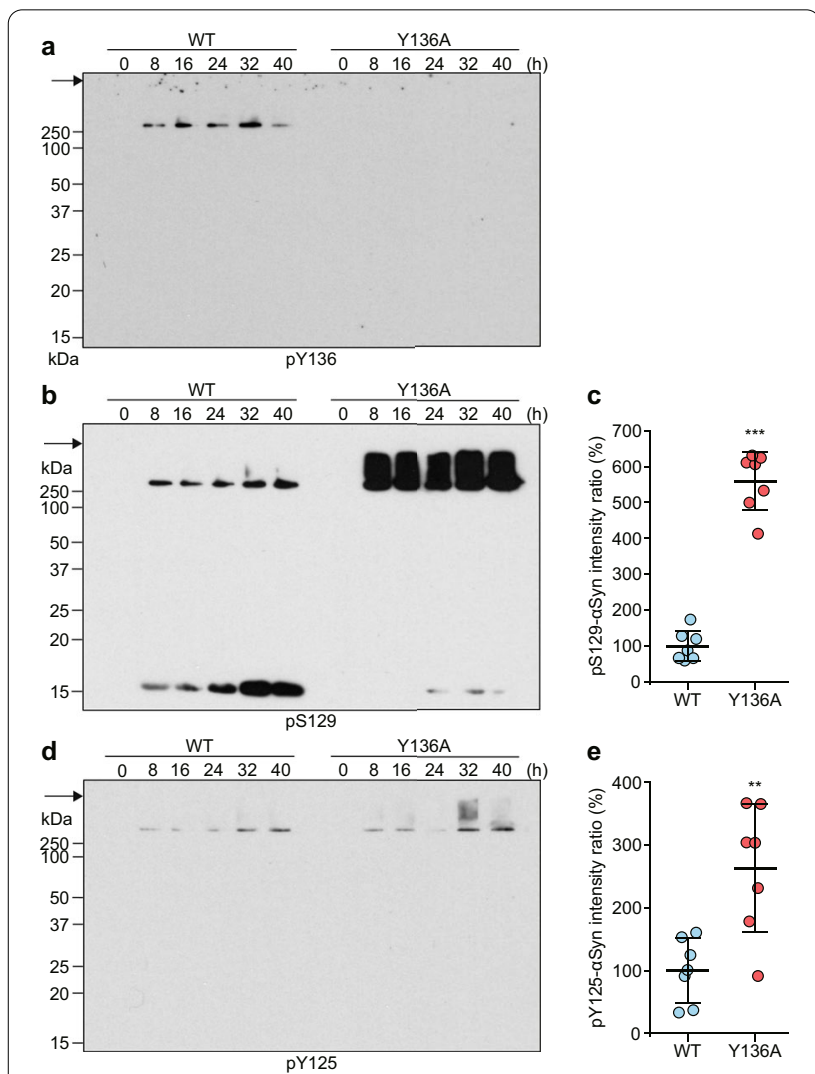

Fig. 6 Blocking Tyr136 phosphorylation promotes phosphorylation of aSyn at Ser129 and Tyr125 induced by CK2. WT r-aSyn or Y136A r-aSyn after 0-40 hours of incubation with CK2 and ATP was analyzed by SDS-PAGE followed by immunoblotting with a anti-pY136-aSyn antibody, b anti-pS129-aSyn antibody ab51253, and d anti-pY125-aSyn antibody. Molecular mass markers are indicated in $\mathrm{kDa}$ on the left side of each panel. Arrows indicate the top of the gel. Intensity ratios (\%) of immunoreactive $\mathbf{c}$ pS129-aSyn, the sum of $>250-k D a$ and $16-k D a$ forms, and e $>250-k D a$ pY125-aSyn after $8 \mathrm{~h}$ of incubation were quantified in seven independent experiments. Data are presented as means \pm standard deviation. Statistical significance was determined using the 2-tailed Student's $t$ test. ${ }^{* *} P<0.01,{ }^{* *} P<0.001$ versus WT $r$-aSyn

showed that polymers of $\alpha \mathrm{Syn}>25 \mathrm{kDa}$ in addition to monomers accumulated in cells transfected with WT $r-\alpha$ Syn or Y136A $r-\alpha$ Syn subjected to agitation, whereas no $\alpha$ Syn immunoreactivity was detected in cells transfected with WT $r-\alpha$ Syn or Y136A $r-\alpha$ Syn without agitation (Fig. 7b). The levels of accumulation of $\alpha$ Syn $>25 \mathrm{kDa}$ were significantly higher in cells transfected with agitated Y136A $r-\alpha$ Syn than with agitated WT $r-\alpha$ Syn (Fig. 7c). Furthermore, the levels of $\alpha$ Syn accumulation in cells transfected with agitated WT $r-\alpha$ Syn or Y136A $r-\alpha$ Syn were significantly increased by overexpression of WT $\alpha$ Syn or Y136A $\alpha$ Syn, respectively (Fig. 7d, e), indicating the prion-like seeding activity of agitated WT $r-\alpha$ Syn 


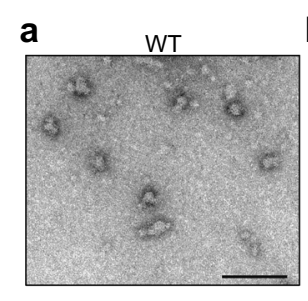

Y136A

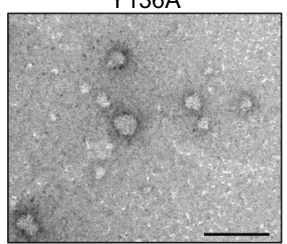

b
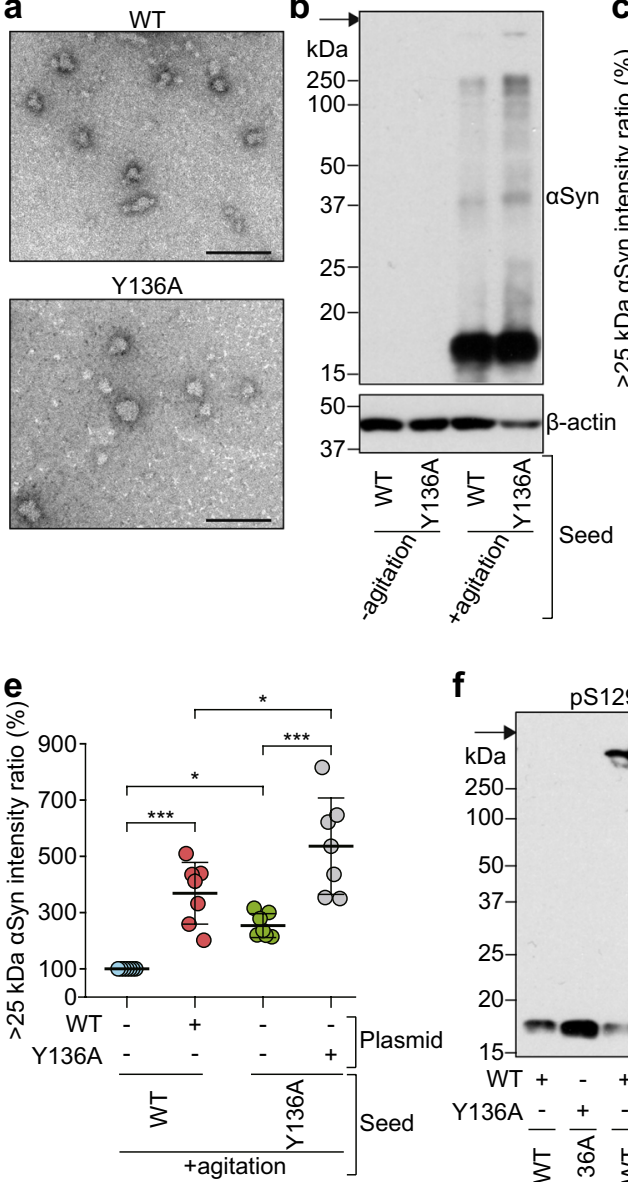

f

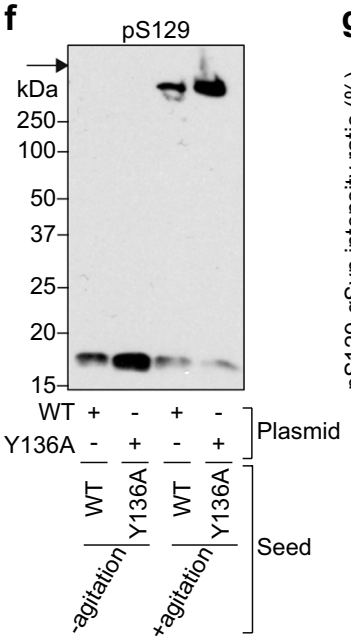

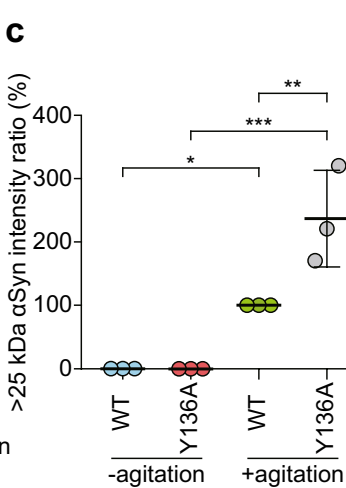

d

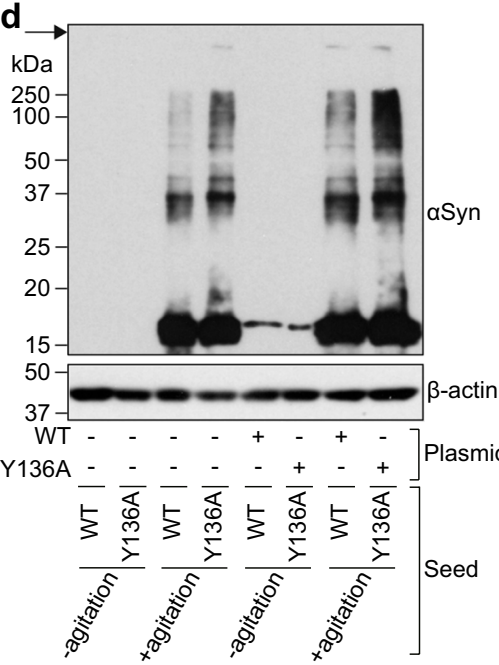

h

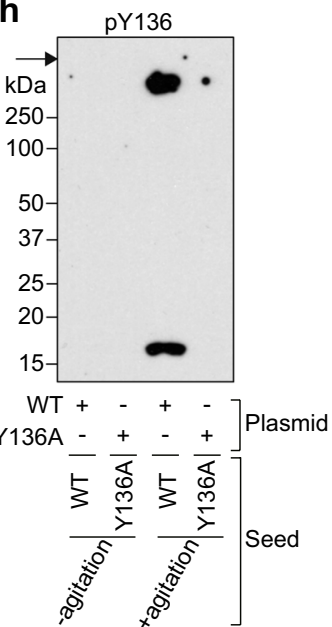

Fig. 7 Tyr136 phosphorylation prevents aggregate formation and Ser129 phosphorylation of aSyn in cultured cells. a WT r-aSyn or Y136A r-aSyn subjected to agitation was examined by TEM. Bars, 100 nm. b-h WT r-aSyn or Y136A r-aSyn seed with (+ agitation) or without (- agitation) agitation was introduced into SH-SY5Y cells with $(+)$ or without $(-)$ pcDNA3.1 plasmid encoding WT or Y136A aSyn. The lysates from cells were analyzed by SDS-PAGE followed by immunoblotting with $\mathbf{b}$, $\mathbf{d}$ anti-aSyn antibody Syn204, $\mathbf{b}$, d anti- $\beta$-actin antibody, $\mathbf{f}$ anti-pS129-aSyn antibody D1R1R, and $\mathbf{h}$ anti-pY136-aSyn antibody. Molecular mass markers are indicated in kDa on the left side of each panel. Arrows indicate the top of the gel. Intensity ratios (\%) of immunoreactive (c, e) aSyn > $25 \mathrm{kDa}$ and $\mathbf{g}$ pS129-aSyn were quantified in at least three independent experiments. Data are presented as means \pm standard deviation. Statistical significance was determined using $\mathbf{c}$, e one-way ANOVA followed by Tukey-Kramer test and g 2-tailed Student's $t$ test. ${ }^{*} P<0.05,{ }^{* *} P<0.01,{ }^{* * *} P<0.001$

and Y136A $r-\alpha$ Syn in cultured cells. Detergent-insoluble aggregates $>250 \mathrm{kDa}$ were detected in cells overexpressing WT $\alpha$ Syn or Y136A $\alpha$ Syn transfected with agitated, but not non-agitated, WT r- $\alpha$ Syn or Y136A r- $\alpha$ Syn using an antibody against pS129- $\alpha$ Syn, although $16-\mathrm{kDa}$ monomer was detected in all samples (Fig. 7f). The intensity of the pS129- $\alpha$ Syn-positive band was significantly higher in cells transfected with agitated Y136A $r-\alpha$ Syn than with agitated WT $r-\alpha$ Syn (Fig. $7 g$ ). Immunostaining with an antibody against pY136- $\alpha$ Syn detected high molecular weight bands at $>250 \mathrm{kDa}$ in addition to monomers only in WT $\alpha$ Syn-overexpressing cells transfected with agitated WT r- $\alpha$ Syn (Fig. 7h). Although antibodies against pY125- $\alpha$ Syn and pY133- $\alpha$ Syn also detected bands in the mass range of around $15-100 \mathrm{kDa}$ in cells, the intensities of the bands were unaffected by the introduction of WT $r-\alpha$ Syn or Y136A $r-\alpha$ Syn regardless of the form of seeds (i.e., agitated or non-agitated) (Additional file 1: Fig. S12). These results suggest that oligomeric $\alpha$ Syn can convert native $\alpha$ Syn into insoluble aggregates that undergo phosphorylation of S129 and Y136, and that blocking Y136 
phosphorylation facilitates aggregate formation and S129 phosphorylation of $\alpha$ Syn in cultured cells.

\section{CK2 inhibitor suppresses aSyn aggregate formation and S129 phosphorylation, and increases Y136 phosphorylation}

To examine whether CK2 is involved in aggregate formation and $C$-terminal phosphorylation of $\alpha$ Syn in cells, we investigated the effects of the CK2 inhibitor, 4,5,6,7-tetrabromobenzotriazole (TBB), in SH-SY5Y cells exposed to agitated WT $r-\alpha$ Syn. TBB significantly reduced the levels of accumulation of $\alpha \operatorname{Syn}>25 \mathrm{kDa}$ in a dose-dependent manner in cells transfected with agitated WT $r-\alpha$ Syn (Fig. 8a, b). TBB treatment also significantly reduced the intensity of the pS129- $\alpha$ Syn-positive band of $\alpha$ Syn aggregates $>250 \mathrm{kDa}$ in the cells in a dose-dependent manner (Fig. 8c, d). Unexpectedly, the cells also showed an increase in the intensity of the pY136- $\alpha$ Syn-positive band of $\alpha$ Syn aggregates $>250 \mathrm{kDa}$ with TBB treatment (Fig. 8e). The levels of pY136- $\alpha$ Syn were significantly increased by TBB in a dose-dependent manner (Fig. 8f). These results suggest that inhibition of CK2 suppresses $\alpha$ Syn aggregate formation by reduction of S129 phosphorylation and an unexpected increase in Y136 phosphorylation in cells.

\section{Discussion}

The results of the present study showed that insoluble aSyn is highly phosphorylated at both Y136 and S129 in the LBD brain. In addition, pY136- $\alpha$ Syn, which was presumed to be oligomeric and/or ubiquitinated, was found to be constitutively expressed in the brain regardless of the presence or absence of neocortical LB. Both pY125- $\alpha$ Syn and pY133- $\alpha$ Syn were almost undetectable by immunoblotting and were detected at low levels by immunohistochemical analysis, indicating that phosphorylated $\alpha$ Syn is present in relatively small amounts in the brain. Moreover, Y125 and Y133 phosphorylation were unaffected by the formation of insoluble $\alpha$ Syn aggregates in the brain. The level of pY125 was reported to be higher in the control than LBD brain by immunoblotting, and was shown to be reduced in the aging human brain [7]. Both control subjects and LBD patients were 65-86 years old in this previous study [7], while brain tissues from patients 75-92 years of age were examined in the present study. The previous study also showed that the level of pY125 in heads from flies expressing WT human $\alpha$ Syn decreased with increasing incubation time at room temperature [7]. Therefore, the lack of detectable pY125 in the brain by immunoblotting was likely due to the age of the patients and the postmortem interval in the present study. Brain tissues were examined 8-12 years postmortem in this study, while the postmortem interval in the

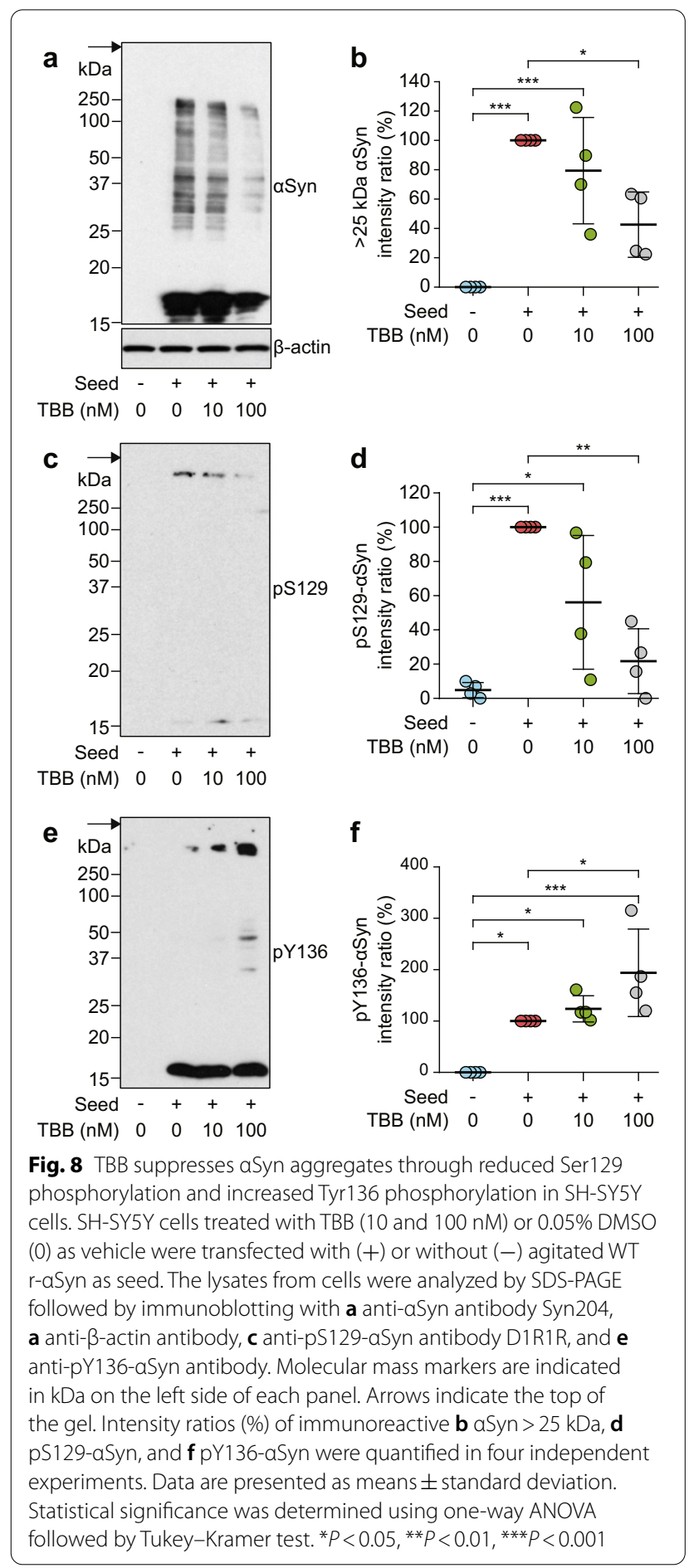

previous study was not clearly indicated. Although all tissues were stored at $-80{ }^{\circ} \mathrm{C}$ until use in this study, the postmortem interval or freeze-thawing may have been involved in the lack of detectable pY125.

Several protein kinases have been suggested to be responsible for S129 phosphorylation of $\alpha$ Syn, including 
CK1, G protein-coupled receptor kinases (GRKs), and polo-like kinases (PLKs). We also showed that CK2 phosphorylated $\alpha$ Syn at S129 in vitro, which was strongly correlated with $\alpha$ Syn aggregate formation consistent with our previous report [29]. Although CK2 has generally been classified as a serine/threonine protein kinase, several studies have demonstrated its tyrosine phosphorylation activity, suggesting that it acts as a dual-specificity kinase $[3,33,37]$. Indeed, we found that the C-terminal tyrosine residues surrounding S129, Y125 and Y136, were phosphorylated by $\mathrm{CK} 2$, and that tyrosine phosphorylation was exclusively found in insoluble $\alpha$ Syn species in vitro. In addition, insoluble $\alpha$ Syn aggregation was accompanied by pY136, but not pY125, as well as pS129 in cultured human cells that had taken up extracellular $\alpha$ Syn oligomers as seeds. These findings suggested that pY136 is related in some way to the formation and propagation of $\alpha$ Syn aggregates in cells. Phosphorylation at certain residues of $\alpha$ Syn has been shown to affect subsequent phosphorylation events in neighboring residues. Mutation of Y125 to phenylalanine (Y125F), preventing phosphorylation at this site, has been shown to decrease the levels of S129 phosphorylation by CK1 in vitro [16]. Double mutation of Y133 and Y136 to phenylalanine enhanced phosphorylation of Y125 by Lyn tyrosine kinase in vitro [22]. In our in vitro experiment, prevention of S129 phosphorylation in S129A mutant blocked Y125 and Y136 phosphorylation by CK2 with formation of $\alpha$ Syn aggregates, suggesting that pS129 mediates phosphorylation of Y125 and Y136 and this plays a role in $\alpha$ Syn aggregate formation. Prominent pS129 was seen within 1 day of incubation of WT $\alpha$ Syn with CK2, while pY125 and pY136 were observed after 7 and 3 days of incubation, respectively. Analysis of the kinetic parameters for phosphorylation of tyrosine-containing peptides by CK2 in vitro demonstrated that tyrosine phosphorylation is less favorable than serine/threonine phosphorylation [20]. Therefore, these results suggest that CK2 preferentially catalyzes phosphorylation of S129, which is essential for subsequent Y125 and Y136 phosphorylation in $\alpha$ Syn.

The substrate specificity of CK2 is determined by one or more negatively charged residues, i.e., aspartic acid (D)/ glutamic acid (E), surrounding the phosphorylatable serine (S) and threonine $(\mathrm{T})$ residues. The minimum consensus sequence is $\mathrm{S} / \mathrm{T}-\mathrm{X}-\mathrm{X}-\mathrm{D} / \mathrm{E}$, where $\mathrm{X}$ can be any amino acid. The most crucial acidic residue position for susceptibility to phosphorylation by CK2 is $n+3$ followed by $n+1$ [21] . In the case of $\alpha$ Syn, the amino acid at position $n+1$ from S129 is E130 (Fig. 1a), which is predicted to mostly act as a specificity determinant for S129 phosphorylation by CK2. Little is known about the substrate specificity of CK2 for tyrosine phosphorylation. If the consensus sequence is commonly recognized by CK2 for tyrosine phosphorylation, it is possible that $\mathrm{Y} 136$ is the C-terminal tyrosine residue most susceptible to phosphorylation, consistent with our results, because the amino acid at positions $\mathrm{n}+1$ and $\mathrm{n}+3$ from Y136 are the acidic residues, E137 and E139, respectively, while acidic residues are present at positions $\mathrm{n}+1$ from Y125 and $\mathrm{n}+2$ from Y133, (E126 and D135, respectively) (Fig. 1a). Two members of the PLK family, PLK2 and PLK3, recognize an acidic residue similar to CK2 [27]. An in vitro study showed that PLK2, and to a lesser extent PLK3, phosphorylated S129 more efficiently than CK2 [28]. However, PLK2 knockout (KO) mice showed not over $50 \%$ decrease in pS129, while PLK3 $\mathrm{KO}$ had little effect on pS129 in various brain regions [4]. The remaining pS129 levels were not reduced by treatment with PLK1-3 inhibitor in PLK2 KO mice [4]. The results suggest that S129 can be phosphorylated by multiple kinases in vivo. Moreover, PLK2 KO has been reported to have no effect on pS129 in LB but not presynaptic terminals in mice [36]. Therefore, it is likely that other, non-PLK kinases, including CK2, mediate phosphorylation of S129 of $\alpha$ Syn aggregates in vivo. Although it remains unclear why pS129 is essential for subsequent tyrosine phosphorylation, pS129 increases the negative charge on the C-terminus by the addition of a $\mathrm{PO}_{4}{ }^{2-}$ group and may therefore lower the threshold for tyrosine phosphorylation by CK2. It has been reported that most of the phosphorylation sites are located in intrinsically disordered regions [12], and that phosphorylation induces folding of the intrinsically disordered protein $[2,18]$. Indeed, phosphorylation of the papillomavirus E2 protein by CK2 has been reported to induce a conformational change that leads to degradation of the protein [25]. It has been also reported that pS129 increases the conformational flexibility of $\alpha$ Syn [24]. Therefore, it is possible that disorder-to-order conformational transitions occur in the intrinsically disordered C-terminal region of $\alpha$ Syn by $\mathrm{pS} 129$, and the conformational changes also enable phosphorylation of tyrosine residues by CK2 in addition to induction of $\alpha$ Syn aggregate formation. The affinity of anti-pS129- $\alpha$ Syn antibody could be enhanced by Y136A mutation, or the levels of pS129 may be increased by Y136A substitution itself. However, the levels of expression of $\alpha$ Syn and the levels of pS129 were not significantly affected by Y136A mutation in $\alpha$ Syn-overexpressing cells as determined by immunoblotting analysis (Additional file 1: Fig. S13). There were also no differences in the expression pattern of $\alpha$ Syn between WT and Y136A $\alpha$ Synoverexpressing cells (Additional file 1: Fig. S13). Moreover, insoluble aggregates and amyloid fibrils of nonphosphorylated Y136A $r-\alpha$ Syn were not formed by incubation (Fig. $5 \mathrm{c}$, Additional file 1: Fig. S7a). Therefore, it is unlikely that anti-pS129- $\alpha$ Syn antibody binds Y136A $\alpha$ Syn with different affinity than WT $\alpha$ Syn and that the increase in pS129 
and acceleration of aggregate formation of $\alpha$ Syn by Y136A mutation are due to Y136A substitution itself. Although the $16-\mathrm{kDa}$ monomer of WT $\alpha$ Syn was phosphorylated at Y136, there was no significant difference in the level of pS129 between WT and Y136A $\alpha$ Syn-overexpressing cells (Additional file 1: Fig. S13). These results suggest that pY136 has little effect on pS129 of $\alpha$ Syn monomer in nondiseased cells.

Preventing phosphorylation of Y136 by Y136A mutation facilitated aggregate formation and S129 phosphorylation of $r-\alpha$ Syn and $\alpha$ Syn in cultured cells. Y136 may also be one of the major phosphorylatable sites for CK2 in negatively charged $\alpha$ Syn aggregates formed with increased pS129 and to protect against further S129 phosphorylation and $\alpha$ Syn aggregate formation by undergoing phosphorylation instead of S129. TBB significantly inhibited S129 phosphorylation and suppressed $\alpha$ Syn aggregate formation in cultured cells. These results suggested that CK2 is the main protein kinase for S129 phosphorylation of $\alpha$ Syn and that phosphorylation of S129 by CK2 is closely related to the formation of $\alpha$ Syn aggregates in SH-SY5Y cells. Therefore, CK2 may be a therapeutic target for LB disease. Unexpectedly, phosphorylation of Y136 in $\alpha$ Syn aggregates was significantly increased by TBB in cultured cells, suggesting that CK2 plays an inhibitory role against Y136 phosphorylation in cells. As CK2 phosphorylates hundreds of physiological substrates to control various cellular processes [5], its in vitro and in vivo functions may not be consistent. CK2 has been reported to phosphorylate threonine residues of Src family tyrosine kinases, thereby resulting in reduction of their activities in vitro [38]. Therefore, the significant increase in pY136 in $\alpha$ Syn aggregates may be due to disinhibition of tyrosine kinases under conditions where CK2 is inhibited in cells, although the major protein kinases for phosphorylation of Y136 have yet to be elucidated, and may contribute to reduction in pS129 and confer protection against $\alpha$ Syn aggregate formation.

\section{Conclusions}

The findings of the present study provide the first evidence that CK2 phosphorylates Y136 in $\alpha$ Syn aggregates by mediating S129 phosphorylation as a dual-specificity kinase and that pY136 has a protective effect against $\alpha$ Syn aggregation. Although the primary kinase for pY136 in vivo is not yet clear, this study suggested that CK2 is a candidate kinase responsible for pY136 and that it participates in regulating $\alpha$ Syn aggregate formation. Further studies are necessary to elucidate the potential roles of the interactions between CK2 and $\alpha$ Syn C-terminal phosphorylation, their involvement in the pathogenesis of LB diseases, and their potential for the development of novel therapeutic strategies.

\section{Abbreviations}

AD: Alzheimer's disease; CK1: Casein kinase 1; CK2: Casein kinase 2; CJD: Creutzfeldt-Jakob disease; LB: Lewy body; LBD: Lewy body dementia; LC-MS/ MS: Liquid chromatography-ion trap mass spectrometry; MS: Mass spectrometry; PD: Parkinson's disease; TBB: 4,5,6,7-Tetrabromobenzotriazole; TEM: Transmission electron microscopy; ThT: Thioflavin T; aSyn: a-Synuclein; WT: Wild-type.

\section{Supplementary Information}

The online version contains supplementary material available at https://doi. org/10.1186/s40478-021-01281-9.

Additional file1. Supplementary fgures.

\section{Acknowledgements}

We thank Fuyuki Kametani of Tokyo Metropolitan Institute of Medical Science for LC-MS/MS analysis, Shinya Dohgu of Fukuoka University for providing SH-SY5Y cells, and Megumi Saiki, Kaori Hirakawa, Shota Hasegawa, Yuhei Moriyama, Saki Ogami, Haruka Taketomi, Yumiko Hori, Kasumi Kubo, Manami Noda, Dan Hokama, Ayumi Yamada, and Hinako Nonaka of Fukuoka University for technical assistance.

\section{Authors' contributions}

KS and KM designed the project. YI and KS collected clinical specimens. YI performed immunohistochemistry. $Y Y, K I, M H$, and $K S$ performed in vitro experiments and cell culture experiments. KS and KS performed immunoblotting of clinical specimens. YI, KS, KM, and KS analyzed the data. KS wrote the manuscript. All authors read and approved the final manuscript.

\section{Funding}

This work was supported by a grant-in-aid for Scientific Research (C) (grant no. 19K07858) from the Ministry of Education, Culture, Sports, Science, and Technology of Japan.

\section{Availability of data and materials}

All data generated or analyzed during this study are included in this published article and its supplementary information files.

\section{Declarations}

Ethics approval and consent to participate

The study protocol was approved by the Institutional Review Board-Independent Ethics Committee of Fukuoka University (ID: 20-03-M2), the Ethics Committee of Nagasaki University (ID: 19083005-2) and the Ethics Committee of Aichi Medical University (ID: 15-017). Informed consent was obtained from the patients and/or their families.

\section{Consent for publication}

Consent for the use of the brain tissue for research purposes and for publication was obtained from the patients and/or their families.

\section{Competing interests \\ The authors report no competing interests.}

\section{Author details}

${ }^{1}$ Department of Physiology and Pharmacology, Faculty of Pharmaceutical Sciences, Fukuoka University, 8-19-1 Nanakuma, Jonan-ku, Fukuoka 814-0180, Japan. ${ }^{2}$ Department of Neuropathology, Institute for Medical Science of Aging, Aichi Medical University, Aichi 480-1195, Japan. ${ }^{3}$ Department of Immunological and Molecular Pharmacology, Faculty of Pharmaceutical Sciences, Fukuoka University, Fukuoka 814-0180, Japan. ${ }^{4}$ Department of Health Sciences, Unit of Medical and Dental Sciences, Nagasaki University Graduate School of Biomedical Sciences, Nagasaki 852-8523, Japan.

Received: 31 July 2021 Accepted: 22 October 2021

Published online: 12 November 2021 


\section{References}

1. Anderson JP, Walker DE, Goldstein JM, de Laat R, Banducci K, Caccavello RJ, Barbour R, Huang J, Kling K, Lee M et al (2006) Phosphorylation of Ser-129 is the dominant pathological modification of alpha-synuclein in familial and sporadic Lewy body disease. J Biol Chem 281:29739-29752. https://doi.org/10.1074/jbc.M600933200

2. Bah A, Vernon RM, Siddiqui Z, Krzeminski M, Muhandiram R, Zhao C, Sonenberg N, Kay LE, Forman-Kay JD (2015) Folding of an intrinsically disordered protein by phosphorylation as a regulatory switch. Nature 519:106-109. https://doi.org/10.1038/nature13999

3. Basnet H, Su XB, Tan Y, Meisenhelder J, Merkurjev D, Ohgi KA, Hunter T, Pillus L, Rosenfeld MG (2014) Tyrosine phosphorylation of histone H2A by CK2 regulates transcriptional elongation. Nature 516:267-271. https://doi. org/10.1038/nature13736

4. Bergeron M, Motter R, Tanaka P, Fauss D, Babcock M, Chiou SS, Nelson S, San Pablo F, Anderson JP (2014) In vivo modulation of polo-like kinases supports a key role for PLK2 in Ser129 a-synuclein phosphorylation in mouse brain. Neuroscience 256:72-82. https://doi.org/10.1016/j.neuro science.2013.09.061

5. Borgo C, D'Amore C, Sarno S, Salvi M, Ruzzene M (2021) Protein kinase CK2: a potential therapeutic target for diverse human diseases. Signal Transduct Target Ther 6:183. https://doi.org/10.1038/s41392-021-00567-7

6. Chen L, Feany MB (2005) Alpha-synuclein phosphorylation controls neurotoxicity and inclusion formation in a Drosophila model of Parkinson disease. Nat Neurosci 8:657-663. https://doi.org/10.1038/nn1443

7. Chen L, Periquet M, Wang X, Negro A, McLean PJ, Hyman BT, Feany MB (2009) Tyrosine and serine phosphorylation of alpha-synuclein have opposing effects on neurotoxicity and soluble oligomer formation. J Clin Investig 119:3257-3265. https://doi.org/10.1172/jci39088

8. Ellis CE, Schwartzberg PL, Grider TL, Fink DW, Nussbaum RL (2001) alphasynuclein is phosphorylated by members of the Src family of proteintyrosine kinases. J Biol Chem 276:3879-3884. https://doi.org/10.1074/jbc. M010316200

9. Fayyad M, Erskine D, Majbour NK, Vaikath NN, Ghanem SS, Sudhakaran IP, Abdesselem H, Lamprokostopoulou A, Vekrellis K, Morris CM et al (2020) Investigating the presence of doubly phosphorylated a-synuclein at tyrosine 125 and serine 129 in idiopathic Lewy body diseases. Brain Pathol. (Zurich, Switz.) 30:831-843. https://doi.org/10.1111/bpa.12845

10. Fujiwara H, Hasegawa M, Dohmae N, Kawashima A, Masliah E, Goldberg MS, Shen J, Takio K, Iwatsubo T (2002) alpha-Synuclein is phosphorylated in synucleinopathy lesions. Nat Cell Biol 4:160-164. https://doi.org/10. 1038/ncb748

11. Hejjaoui M, Butterfield S, Fauvet B, Vercruysse F, Cui J, Dikiy I, Prudent M, Olschewski D, Zhang Y, Eliezer D et al (2012) Elucidating the role of C-terminal post-translational modifications using protein semisynthesis strategies: a-synuclein phosphorylation at tyrosine 125. J Am Chem Soc 134:5196-5210. https://doi.org/10.1021/ja210866j

12. lakoucheva LM, Radivojac P, Brown CJ, O'Connor TR, Sikes JG, Obradovic Z, Dunker AK (2004) The importance of intrinsic disorder for protein phosphorylation. Nucleic Acids Res 32:1037-1049. https://doi.org/10.1093/ nar/gkh253

13. Ishii A, Nonaka T, Taniguchi S, Saito T, Arai T, Mann D, Iwatsubo T, Hisanaga S, Goedert M, Hasegawa M (2007) Casein kinase 2 is the major enzyme in brain that phosphorylates Ser129 of human alpha-synuclein: Implication for alpha-synucleinopathies. FEBS Lett 581:4711-4717. https://doi.org/10. 1016/j.febslet.2007.08.067

14. Karampetsou M, Ardah MT, Semitekolou M, Polissidis A, Samiotaki M, Kalomoiri M, Majbour N, Xanthou G, El-Agnaf OMA, Vekrellis K (2017) Phosphorylated exogenous alpha-synuclein fibrils exacerbate pathology and induce neuronal dysfunction in mice. Sci Rep 7:16533. https://doi. org/10.1038/s41598-017-15813-8

15. Kiely AP, Asi YT, Kara E, Limousin P, Ling H, Lewis P, Proukakis C, Quinn N, Lees AJ, Hardy J et al (2013) a-Synucleinopathy associated with G51D SNCA mutation: a link between Parkinson's disease and multiple system atrophy? Acta Neuropathol 125:753-769. https://doi.org/10.1007/ s00401-013-1096-7

16. Kosten J, Binolfi A, Stuiver M, Verzini S, Theillet FX, Bekei B, van Rossum M, Selenko P (2014) Efficient modification of alpha-synuclein serine 129 by protein kinase $\mathrm{CK} 1$ requires phosphorylation of tyrosine 125 as a priming event. ACS Chem Neurosci 5:1203-1208. https://doi.org/10.1021/cn500 2254
17. Lee G, Tanaka M, Park K, Lee SS, Kim YM, Junn E, Lee SH, Mouradian MM (2004) Casein kinase II-mediated phosphorylation regulates alpha-synuclein/synphilin-1 interaction and inclusion body formation. J Biol Chem 279:6834-6839. https://doi.org/10.1074/jbc.M312760200

18. Liu N, Guo Y, Ning S, Duan M (2020) Phosphorylation regulates the binding of intrinsically disordered proteins via a flexible conformation selection mechanism. Commun Chem 3:123. https://doi.org/10.1038/ s42004-020-00370-5

19. Mahul-Mellier AL, Fauvet B, Gysbers A, Dikiy I, Oueslati A, Georgeon S, Lamontanara AJ, Bisquertt A, Eliezer D, Masliah E et al (2014) c-Abl phosphorylates a-synuclein and regulates its degradation: implication for a-synuclein clearance and contribution to the pathogenesis of Parkinson's disease. Hum Mol Genet 23:2858-2879. https://doi.org/10.1093/ hmg/ddt674

20. Marin O, Meggio F, Sarno S, Cesaro L, Pagano MA, Pinna LA (1999) Tyrosine versus serine/threonine phosphorylation by protein kinase casein kinase-2. A study with peptide substrates derived from immunophilin Fpr3. J Biol Chem 274:29260-29265. https://doi.org/10.1074/jbc.274.41. 29260

21. Meggio F, Pinna LA (2003) One-thousand-and-one substrates of protein kinase CK2? FASEB J: Off Publ Feder Am Soc Exp Biol 17:349-368. https:// doi.org/10.1096/fi.02-0473rev

22. Negro A, Brunati AM, Donella-Deana A, Massimino ML, Pinna LA (2002) Multiple phosphorylation of alpha-synuclein by protein tyrosine kinase Syk prevents eosin-induced aggregation. FASEB J: Off Publ Feder Am Soc Exp Biol 16:210-212. https://doi.org/10.1096/fj.01-0517fje

23. Okochi M, Walter J, Koyama A, Nakajo S, Baba M, Iwatsubo T, Meijer L, Kahle PJ, Haass C (2000) Constitutive phosphorylation of the Parkinson's disease associated alpha-synuclein. J Biol Chem 275:390-397. https://doi. org/10.1074/jbc.275.1.390

24. Paleologou KE, Schmid AW, Rospigliosi CC, Kim HY, Lamberto GR, Fredenburg RA, Lansbury PT Jr, Fernandez CO, Eliezer D, Zweckstetter M et al (2008) Phosphorylation at Ser-129 but not the phosphomimics S129E/D inhibits the fibrillation of alpha-synuclein. J Biol Chem 283:16895-16905. https://doi.org/10.1074/jbc.M800747200

25. Penrose KJ, Garcia-Alai M, de Prat-Gay G, McBride AA (2004) Casein Kinase Il phosphorylation-induced conformational switch triggers degradation of the papillomavirus E2 protein. J Biol Chem 279:22430-22439. https:// doi.org/10.1074/jbc.M314340200

26. Ryu MY, Kim DW, Arima K, Mouradian MM, Kim SU, Lee G (2008) Localization of CKII beta subunits in Lewy bodies of Parkinson's disease. J Neurol Sci 266:9-12. https://doi.org/10.1016/j.jns.2007.08.027

27. Salvi M, Trashi E, Cozza G, Franchin C, Arrigoni G, Pinna LA (2012) Investigation on PLK2 and PLK3 substrate recognition. Biochem Biophys Acta 1824:1366-1373. https://doi.org/10.1016/j.bbapap.2012.07.003

28. Salvi M, Trashi E, Marin O, Negro A, Sarno S, Pinna LA (2012) Superiority of PLK-2 as a-synuclein phosphorylating agent relies on unique specificity determinants. Biochem Biophys Res Commun 418:156-160. https://doi. org/10.1016/j.bbrc.2011.12.152

29. Sano K, Atarashi R, Satoh K, Ishibashi D, Nakagaki T, Iwasaki Y, Yoshida M, Murayama S, Mishima K, Nishida N (2018) Prion-Like Seeding Of Misfolded a-synuclein in the brains of dementia with lewy body patients in RT-QUIC. Mol Neurobiol 55:3916-3930. https://doi.org/10.1007/ s12035-017-0624-1

30. Schreurs S, Gerard M, Derua R, Waelkens E, Taymans JM, Baekelandt V, Engelborghs $Y$ (2014) In vitro phosphorylation does not influence the aggregation kinetics of WT a-synuclein in contrast to its phosphorylation mutants. Int J Mol Sci 15:1040-1067. https://doi.org/10.3390/ijms150110 40

31. Schwab C, DeMaggio AJ, Ghoshal N, Binder LI, Kuret J, McGeer PL (2000) Casein kinase 1 delta is associated with pathological accumulation of tau in several neurodegenerative diseases. Neurobiol Aging 21:503-510. https://doi.org/10.1016/s0197-4580(00)00110-x

32. Smith WW, Margolis RL, Li X, Troncoso JC, Lee MK, Dawson VL, Dawson TM, Iwatsubo T, Ross CA (2005) Alpha-synuclein phosphorylation enhances eosinophilic cytoplasmic inclusion formation in $\mathrm{SH}-\mathrm{SY} 5 \mathrm{Y}$ cells. J Neurosci: Off J Soc Neurosci 25:5544-5552. https://doi.org/10.1523/jneur osci.0482-05.2005

33. Vilk G, Weber JE, Turowec JP, Duncan JS, Wu C, Derksen DR, Zien P, Sarno S, Donella-Deana A, Lajoie G et al (2008) Protein kinase CK2 catalyzes 
tyrosine phosphorylation in mammalian cells. Cell Signal 20:1942-1951. https://doi.org/10.1016/j.cellsig.2008.07.002

34. Wakamatsu M, Ishii A, Ukai Y, Sakagami J, Iwata S, Ono M, Matsumoto K, Nakamura A, Tada N, Kobayashi Ket al (2007) Accumulation of phosphorylated alpha-synuclein in dopaminergic neurons of transgenic mice that express human alpha-synuclein. J Neurosci Res 85:1819-1825. https://doi. org/10.1002/jnr.21310

35. Waxman EA, Giasson BI (2008) Specificity and regulation of casein kinasemediated phosphorylation of alpha-synuclein. J Neuropathol Exp Neurol 67:402-416. https://doi.org/10.1097/NEN.0b013e31816fc995

36. Weston LJ, Stackhouse TL, Spinelli KJ, Boutros SW, Rose EP, Osterberg VR, Luk KC, Raber J, Weissman TA, Unni VK (2021) Genetic deletion of Polo-like kinase 2 reduces alpha-synuclein serine-129 phosphorylation in presynaptic terminals but not Lewy bodies. J Biol Chem 296:100273. https://doi.org/10.1016/j.jbc.2021.100273

37. Wilson LK, Dhillon N, Thorner J, Martin GS (1997) Casein kinase II catalyzes tyrosine phosphorylation of the yeast nucleolar immunophilin Fpr3. J Biol Chem 272:12961-12967. https://doi.org/10.1074/jbc.272.20.12961

38. Yokoyama T, Kamata Y, Ohtsuki K (2004) Casein kinase 2 (CK2)-mediated reduction of the activities of Src family tyrosine kinases in vitro. Biol Pharm Bull 27:1895-1899. https://doi.org/10.1248/bpb.27.1895

\section{Publisher's Note}

Springer Nature remains neutral with regard to jurisdictional claims in published maps and institutional affiliations.
Ready to submit your research? Choose BMC and benefit from:

- fast, convenient online submission

- thorough peer review by experienced researchers in your field

- rapid publication on acceptance

- support for research data, including large and complex data types

- gold Open Access which fosters wider collaboration and increased citations

- maximum visibility for your research: over 100M website views per year

At BMC, research is always in progress.

Learn more biomedcentral.com/submissions 